\title{
Bright Lyman- $\alpha$ emitters among Spitzer SMUVS galaxies in the MUSE/COSMOS field ${ }^{\star}$
}

\author{
G. Rosani ${ }^{1}$, G. B. Caminha ${ }^{1}$, K. I. Caputi ${ }^{1,2}$, and S. Deshmukh ${ }^{1}$ \\ ${ }^{1}$ Kapteyn Astronomical Institute, University of Groningen, PO Box 800, 9700 Groningen, The Netherlands \\ e-mail: rosani@astro.rug.nl \\ 2 Cosmic Dawn Center (DAWN), Niels Bohr Institute, University of Copenhagen, Juliane Maries vej 30, 2100 Copenhagen, Denmark
}

Received 26 April 2019 / Accepted 12 November 2019

\begin{abstract}
We search for the presence of bright Ly $\alpha$ emitters among Spitzer SMUVS galaxies at $z>2.9$ making use of homogeneous MUSE spectroscopic data. Although these data only cover a small region of COSMOS, MUSE has the unique advantage of providing spectral information over the entire field, without the need of target pre-selection. This results in an unbiased detection of all the brightest Ly $\alpha$ emitters among the SMUVS sources, which by design are stellar-mass selected galaxies. Within the studied area, $14 \%$ of the SMUVS galaxies at $z>2.9$ have Ly $\alpha$ fluxes $F_{\lambda} \gtrsim 7 \times 10^{-18} \mathrm{erg} \mathrm{s}^{-1} \mathrm{~cm}^{-2}$. These Ly $\alpha$ emitters are characterized by three types of emission, $47 \%$ show a single-line profile, $19 \%$ present a double peak or a blue bump, and $31 \%$ show a red tail. One object (3\%) shows both a blue bump and a red tail. We also investigate the spectral energy distribution (SED) properties of the SMUVS galaxies that are MUSE detected and those that are not. After stellar mass matching the two populations, we find that the MUSE detected galaxies have generally lower extinction than SMUVS-only objects, while there is no clear intrinsic difference in the mass and age distributions of the two samples. For the MUSE-detected SMUVS galaxies, we compare the instantaneous star formation rate lower limit obtained from the Ly $\alpha$ line with its past average derived from SED fitting, and find evidence for rejuvenation in some of our oldest objects. In addition, we study the spectra of those $\operatorname{Ly} \alpha$ emitters that are not detected in SMUVS in the same field. We find that of the emission line profiles shown $67 \%$ have a single line, $3 \%$ a blue bump, and $30 \%$ a red tail. The difference in profile distribution could be ascribed to the fainter Ly $\alpha$ luminosities of the MUSE sources not detected in SMUVS and an intrinsically different mass distribution. Finally, we search for the presence of galaxy associations using the spectral redshifts. The integral coverage of MUSE reveals that these associations are 20 times more likely than what is derived from all the other existing spectral data in COSMOS, which is biased by target pre-selection.
\end{abstract}

Key words. galaxies: high-redshift - galaxies: star formation - cosmology: observations

\section{Introduction}

The Lyman- $\alpha(\operatorname{Ly} \alpha)$ line contains important information about some of the main physical processes occurring in galaxies. In particular, bright Ly $\alpha$ emitters are tracers of the most prominent unobscured star formation activity at different cosmic times. The interpretation of the Ly $\alpha$ line is not trivial, however, because of its resonant nature; it is easily scattered by the interstellar medium (ISM) on its way out of the galaxy. Furthermore, Ly $\alpha$ photons are absorbed by dust and re-emitted at longer wavelengths, thus subtracting them from the line intensity. The kinematics of the gas also needs to be taken into account. All these processes give rise to different line profile shapes depending on the conditions surrounding the emitter galaxy (Shapley et al. 2003; Karman et al. 2014, 2017; Martin et al. 2015; Dijkstra 2017; Hernán-Caballero et al. 2017; Bridge et al. 2018; Erb et al. 2018; Gurung-Lopez et al. 2019; Nakajima et al. 2018; Orlitová et al. 2018; Sobral et al. 2018; Vanzella et al. 2018; Kimm et al. 2019; Marchi et al. 2019; Remolina-Gutiérrez \& Forero-Romero 2019; Smith et al. 2019).

It is possible to recover the original $\operatorname{Ly} \alpha$ flux if information on the $\mathrm{H} \alpha$ line is available. There is no canonical conversion

\footnotetext{
* Full Table A.1 is only available at the CDS via anonymous ftp to cdsarc.u-strasbg. fr (130.79.128.5) or via http://cdsarc. u-strasbg.fr/viz-bin/cat/J/A+A/633/A159
}

factor between $\operatorname{Ly} \alpha$ and $\mathrm{H} \alpha$ because secondary effects influence the conversion, but assuming case $\mathrm{B}$ recombination the values can reasonably range from $\approx 8$ (Dijkstra 2017 ) to $\sim 8.7$ (Hu et al. 1998). When information on the $\mathrm{H} \alpha$ line is not present, we can rely on radiative transfer models exclusively treating the Ly $\alpha$ line to try and recover the original emission from line shape fitting. These models need to take into account the composition of the circumgalactic medium, the presence of dust, how dense the neutral hydrogen is, as well as the gas dynamics and the time evolution of the medium along with the star formation event. Models reproducing the shape of the Ly $\alpha$ emission go from the early approach of Tenorio-Tagle et al. (1999) and Mas-Hesse et al. (2003) to the more recent models by Verhamme et al. (2008, 2018), Gronke (2017), and Kakiichi \& Gronke (2019), among others. Finally, selecting objects using the Ly $\alpha$ line is a way to ensure that the more active star-forming non-dusty galaxies are selected (Zhang et al. 2019).

The use of Spitzer (Werner et al. 2004) data in the 3.6/4.5 $\mu \mathrm{m}$ bands allows us to access the red flat part of the spectrum of highredshift galaxies. This results in a stellar mass selection, as the more luminous high- $z$ galaxies at those wavelengths are typically the most massive. These objects are interesting because they represent possible progenitors of today's most massive galaxies and because they possibly played an integral role in the peak of star formation history around $z \sim 2$ (Caputi et al. 2011; Deshmukh et al. 2018; Martinache et al. 2018). 
By combining the more massive galaxies selected by Spitzer and the most prominent $\operatorname{Ly} \alpha$ emitters, we probe a very specific part of the galaxy evolution picture. We not only select the most massive objects at early times, but we also ensure that they are intensely star-forming and contain relatively little dust by detecting their Ly $\alpha$ emission.

Previous studies combining Ly $\alpha$ information with photometric SED fitting have been able to constrain stellar mass, age, star formation rate (SFR), and $E(B-V)$ of Ly $\alpha$ emitters among other properties. The general result of these studies is that Ly $\alpha$ emitters are young, prominently star-forming, mostly unobscured intermediate-mass galaxies. Some of these studies also find evidence of more massive objects being present among the Ly $\alpha$ population. Moreover, the age distribution of the Ly $\alpha$ emitters presents in some cases an age bimodality, for which a significant fraction of the galaxies studied is not young, but has ages around 1 Gyr (Lai et al. 2008; Finkelstein et al. 2009; Ono et al. 2010; Pentericci et al. 2009, 2010; Yuma et al. 2010; Guaita et al. 2011; Acquaviva et al. 2012; Mallery et al. 2012; Curtis-Lake et al. 2013; McLinden et al. 2014; De Barros et al. 2017; Hao et al. 2018; Marchi et al. 2019).

Among the best studied blank areas of the sky is the COSMOS field (Scoville et al. 2007), for which a wide range of homogeneous and deep data sets are available. One of the surveys spanning part of the field is the Spitzer Matching Survey of the UltraVISTA ultra-deep Stripes (SMUVS, Ashby et al. 2018). The SMUVS survey combines Spitzer 3.6 and $4.5 \mu \mathrm{m}$ data with 26 complementary photometric bands to infer the redshift and physical properties of its galaxies. Most importantly, it uses the Spitzer InfraRed Array Camera (IRAC) data (Fazio et al. 2004) to gain access to the flat part of the continuum of high-redshift objects. The SMUVS survey has already produced a number of studies of galaxy properties at redshift $z>2$ (Caputi et al. 2017; Cowley et al. 2018, 2019; Deshmukh et al. 2018).

The Multi Unit Spectroscopic Explorer spectrograph (MUSE, Bacon et al. 2010) provides a powerful tool to expand the existing analysis of any part of the sky. Being an integral field unit (IFU) spectrograph, it allows to take the spectra of $\mathrm{a} \sim 1^{\prime} \times 1^{\prime}$ portion of the sky with no source pre-selection. Furthermore, new sources can be discovered serendipitously and biases in the galaxy sample, inherent to slit spectroscopy, can be avoided.

As we aim to combine MUSE and SMUVS, and given the wavelength range covered by MUSE, the spectral feature available for us to study in objects above $z \sim 3$ is the Ly $\alpha$ emission. This line emission is visible in the MUSE spectral range for objects at redshifts $2.9 \lesssim z \lesssim 6.6$.

The scope of this paper is to analyze the physical properties of the more prominent Ly $\alpha$ emitters detected in MUSE. We thus use homogeneous MUSE observations in subregions of the COSMOS field of view to spectroscopically confirm SMUVS sources in an area of $\approx 20.79 \operatorname{arcmin}^{2}$. We give special attention to sources in the redshift range $2.9 \leq z \leq 6.6$, where we can obtain secure confirmation from the Ly $\alpha$ line with MUSE, and study its physical properties using the rich broadband photometry from SMUVS, also comparing with the sample of non-Lya emitters at the same redshift range. Furthermore, we also list new sources detected by a blind search performed in the MUSE pointings and test our spectroscopic sample for possible physical associations.

In Sect. 2 we describe the data we used, in Sect. 3 we outline our results, and in Sect. 4 we discuss our results and draw our conclusion. Throughout this paper we adopt a flat $\Lambda \mathrm{CDM}$ cosmology with $H_{0}=69.6 \mathrm{~km} \mathrm{~s}^{-1} \mathrm{Mpc}^{-1}, \Omega_{\mathrm{m}}=0.286$, and $\Omega_{\Lambda}=0.714$.

\section{Data}

The COSMOS field is one of the most observed regions of the sky with numerous ancillary data sets obtained from ground- and space-based observatories. In this section we describe only the data sets that we used for our sample selection.

\subsection{SMUVS sources}

We used the version of the SMUVS catalog (Ashby et al. 2018) presented in Deshmukh et al. (2018) to select part of our sources. We focused on the area of the MUSE/COSMOS GTO field. SMUVS is a Spitzer Space Telescope Exploration Science Program that combines observations in the IRAC (Fazio et al. 2004) $3.6 \mu \mathrm{m}$ and the $4.5 \mu \mathrm{m}$ bands, taken over $\sim 0.66 \mathrm{deg}^{2}$ of the COSMOS field. The area observed by SMUVS overlaps with the three UltraVISTA ultra-deep stripes (McCracken et al. 2012) and with the COSMOS deepest optical coverage of the Subaru telescope (Taniguchi et al. 2007).

The SMUVS source detection is a double-selection in the $H K_{s}$ average stack maps constructed using data from the UltraVISTA third data release and in the 3.6/4.5 $\mu$ m IRAC bands. As described in Deshmukh et al. (2018), SExtractor (Bertin \& Arnouts 1996) is applied on the $H K_{s}$ UltraVISTA maps to select sources that will then be used as priors to perform a point spread function (PSF) fitting on the IRAC images to finalize the selection. The SMUVS catalog includes multi-wavelength photometric data available for COSMOS in 26 bands, from the $U$ through the UltraVISTA $K_{s}$ band. All this photometric data, along with the IRAC photometry, has been used to perform the spectral energy distribution (SED) fitting and derive physical properties for about 300000 galaxies (Deshmukh et al. 2018). In this paper we consider the $\sim 3000$ SMUVS sources that lie on the $20.79 \mathrm{arcmin}^{2}$ area of the MUSE COSMOS/GTO program.

\subsection{MUSE spectroscopy}

In this work we analyze archival data from MUSE (Bacon et al. 2010 ) in the COSMOS field (Scoville et al. 2007), over an area of $20.79 \operatorname{arcmin}^{2}$ embedded in the SMUVS footprint (Ashby et al. 2018). MUSE is one of the latest spectrographs mounted on the Very Large Telescope and offers integral field spectroscopy over an entire $1 \operatorname{arcmin}^{2}$ field of view, providing a spectrum for each $0.2 \times 0.2 \operatorname{arcsec}^{2}$ pixel element. Therefore, the observations do not require pre-selection of targets, and thanks to its small pixel size, the source separation is limited only by observational conditions. MUSE covers the wavelength range 4750-9350 $\AA$ with a spectral bin of $1.25 \AA$ pixel $^{-1}$, resulting in an average resolving power $R \approx 3000$ at $\lambda \sim 7500 \AA$ and an almost constant resolution of $\Delta \lambda \approx 2.4 \AA$.

We made use of a homogeneous data set obtained by the MUSE consortium under the Guaranteed Time Programme IDs 095.A-0240, 096.A-0090, 097.A-0160, and 098.A-0017 (P.I.: L. Wisotzki) as part of the MUSE-Wide survey (Herenz et al. 2017; Diener et al. 2017; Urrutia et al. 2019). The observations consist of 23 different MUSE pointings with one hour of exposure time, of which 21 form a contiguous area in a $3 \times 7$ mosaic, and the remaining two are located in a region $\approx 5$ arcmin apart. The data acquisition was carried out under fair observational conditions with a median seeing of 1 '. 10 , from the DIMM station measurements, and $\approx 17 \%$ of the exposures have seeing higher than $11^{\prime \prime 5}$. The field of view was chosen in order to overlap with deep HST imaging from the CANDELS/COSMOS survey (Grogin et al. 2011; Koekemoer et al. 2011), maximizing 


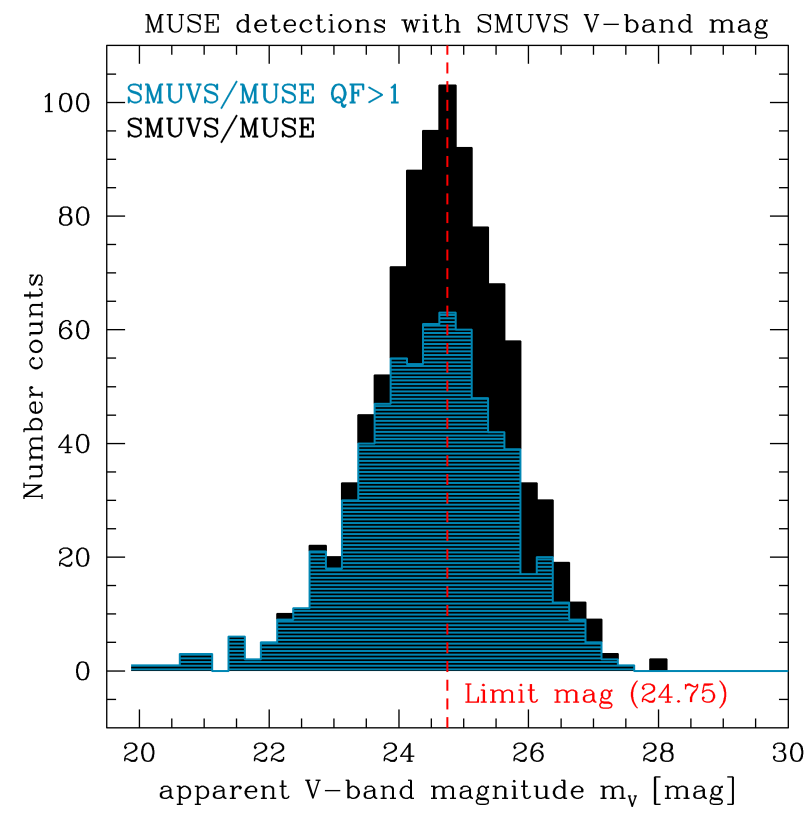

Fig. 1. Histogram of the $V$-band magnitudes assigned by SMUVS to our MUSE detected sources. The peak of the distribution shows the magnitude after which the number counts of MUSE drop significantly.

the amount of photometric information we have on our objects. Additional MUSE pointings from different GO and GTO programs overlapping with the SMUVS field are publicly available. However, we do not consider them here as our aim was to work with a data set of homogeneous depth for the sake of clarity in our results and conclusions.

We retrieved the MUSE raw exposures and calibration files from the ESO archive and used the standard reduction pipeline version 2.0.3 (Weilbacher et al. 2006, 2012, 2014) in combination with the MUSE Python Data Analysis Framework (MPDAF version 2.3, Bacon et al. 2016; Piqueras et al. 2017) and the Zurich Atmosphere Purge (ZAP version 2.1, Soto et al. 2016) to create the final data cubes.

Finally, we corrected the WCS coordinates by using SExtractor to identify the centroids of the brighter objects in the MUSE white images and the CANDELS HST F160W image. We verified that MUSE shows an average offset with respect to HST of $0.141^{\prime \prime}$ with standard deviation $0.110^{\prime \prime}$, while the offset with the reported SMUVS coordinates is $0.196 \pm 0.123^{\prime \prime}$. We corrected the MUSE coordinates taking the HST centroids as reference and note that the offsets between the different catalogs are always smaller than the MUSE pixel scale (0.2 arcsec).

\section{Results}

\subsection{SMUVS sources in the MUSE/COSMOS GTO fields}

We searched for detections of the SMUVS sources in the $20.79 \operatorname{arcmin}^{2}$ covered by the MUSE datacubes, and measure their redshifts from emission and/or absorption lines in their spectra. We consider a SMUVS counterpart detected in MUSE when the MUSE emission arises within $1^{\prime \prime}$ from the SMUVS source centroid. Furthermore, we use HST images from CANDELS to verify possible contamination from nearby sources. We find that the MUSE emission can always be univocally assigned to one source, and we discuss the implications of source contamination on the photometry of our sample in more detail in Sect. 3.3.
As explained above, the advantage of analyzing the MUSE data with respect to any other spectroscopic data set in COSMOS is that MUSE does not require a source pre-selection, and thus the identification completeness is solely governed by the spectroscopic depth. Figure 1 shows that if we translate the depth to a $V$-band magnitude, the number counts in the MUSE pointings start to drop significantly after a magnitude of $\sim 24.75$. We consider this limiting magnitude to compare the depth of the MUSE pointings to the depth of the SMUVS survey.

Out of 2997 SMUVS objects present in the area covered by MUSE, we managed to successfully identify 1038 objects spectroscopically. All redshifts have been measured and agreed upon by two independent observers, following the work philosophy adopted in, for example, the zCOSMOS spectroscopic survey (Lilly et al. 2007).

Also similarly to zCOSMOS, we classify the quality of our spectra by applying the following quality flags (QFs):

- -99: non-detection;

- 0: galactic stars, independently of the spectral quality;

- 1: redshift measurement is only tentative;

- 2: relatively secure redshift measurement, with the spectrum showing faint line(s) and/or a continuum, for which the redshift is likely to be correct;

- 3: very secure redshift measurement, typically based on more than one emission line and/or a clear continuum with absorption lines;

- 4: text-book spectrum with emission and absorption lines, and a very clear continuum;

- 9: redshift based on a single but clearly detected emission line, for which we are unsure about its identification. In these cases, a few alternative spectroscopic redshift values are possible for the source.

The MUSE detection rate for the whole SMUVS sample is $\sim 35 \%$ (=1038/2997), considering all detections regardless of their QF. Among these objects, a total of 691 have a spectroscopic redshift measurement with $\mathrm{QF} \geq 2$, i.e., $\sim 23 \%$ of all the SMUVS sample in the COSMOS/MUSE GTO field, with the following distribution: 49 galaxies have $\mathrm{QF}=2 ; 486$ have $\mathrm{QF}=3 ; 25$ have $\mathrm{QF}=4$; and 131 are classified with $\mathrm{QF}=9$, as shown in the lower panel of Fig. 2. Furthermore, we also detected 41 stars $(\mathrm{QF}=0)$ and 306 galaxies for which the MUSE data quality is not good enough to constrain the redshift of the object with a high enough confidence level $(\mathrm{QF}=1)$. The remaining 1959 SMUVS objects are non-detections in MUSE.

The upper panel of Fig. 2 shows the redshift distribution of our SMUVS/MUSE sources in blue and the $z_{\text {phot }}$ distribution of all the SMUVS sources in the COSMOS/GTO field in red. The SMUVS histogram has been renormalized to match the SMUVS/MUSE sample numbers. As is evident, most of the SMUVS/MUSE detections are located at low redshifts with two overdensities at $z \sim 0.7$ and $z \sim 0.9$, which belong to previously identified large-scale structures in the COSMOS field (Le Fèvre et al. 2005; Kovač et al. 2010). Since the MUSE data is shallow, it is natural that we only see the brighter sources at higher redshifts. We can also see that the MUSE detections clearly identify the overdensities, and favor redshifts where strong emission lines are present in the MUSE wavelength range (i.e., [OII] between $\sim 0.2$ and $\sim 1.4$ and Ly $\alpha$ above $z=2.9$ ). This is in contrast with the photometric redshift distribution, whose intrinsic dispersion smooths out the peaks, making it less suitable to identify preferential redshifts.

Among the SMUVS sources with MUSE QF $\geq 2$ there are 39 with spectroscopic redshift $z_{\text {spec }} \geq 2$. These include three 

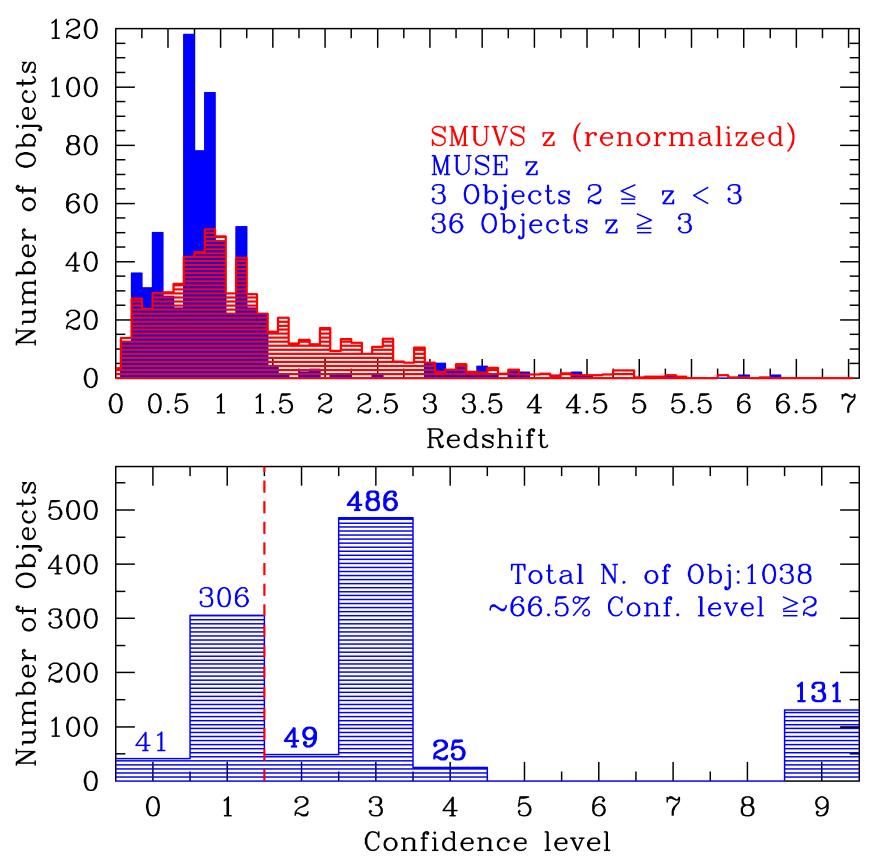

Fig. 2. Upper panel: redshift distribution of our sample of SMUVS/MUSE sources with spectral $\mathrm{QF} \geq 2$ in blue. These objects constitute $\sim 66.5 \%$ of the 1038 SMUVS objects identified with MUSE, and $\sim 23 \%$ of all the SMUVS objects in the COSMOS/MUSE GTO field. The distribution of all the SMUVS redshifts in our MUSE fields is drawn in red and renormalized to the number of MUSE sources detected with $\mathrm{QF} \geq 2$. Lower panel: distribution of spectral $\mathrm{QF}$ for the 1038 MUSE detected sources. The dashed line indicates the boundary between a secure and an uncertain redshift measurement. Quality flag 0 was assigned to all galactic stars, independently of their actual spectral quality.

sources in the redshift range $2 \leq z<3$ and 36 with redshifts $z \geq 3$. The $2 \leq z<3$ sources consist of two bright galaxies with absorption lines and one AGN with broad CIV and [CIII] emission; the $z \geq 3$ sources are prominent Ly $\alpha$ emitters. If we compare the blue and red histograms in Fig. 2, we can see that the MUSE incidence is comparable to SMUVS until $z>4$. For the higher redshift, it is the SMUVS relative incidence that is more pronounced.

At $1.5 \lesssim z \lesssim 3$ the number of identified SMUVS/MUSE objects is drastically lower than at higher redshifts. This is known as the "redshift desert", where no strong nebular emission line falls into the wavelength range covered by MUSE, and thus makes detection particularly difficult. This is clearly illustrated if we look at the number of sources identified in SMUVS in the same redshift range (see red histogram in Fig. 2).

Table 1 shows the results of our redshift measurements for our high-redshift $\left(z_{\mathrm{spec}} \geq 2\right)$ sample. We list the SMUVS ID and position of the objects, as well as the spectroscopic redshifts measured and the quality flag assigned to their spectra. There are three sources with $\mathrm{QF}=2$, additional 20 sources with $\mathrm{QF}=3$, and 16 sources with $\mathrm{QF}=9$. For some of the latter, the ambiguity in the single emission line identification could be solved via the available photometric redshifts of these sources (Deshmukh et al. 2018), as will be discussed in Sect. 3.3. We also note that one of our objects (no. 73761) has a previous spectroscopic redshift identification obtained with MOSFIRE on the Keck Telescope (Kriek et al. 2015), and that our own redshift is in good agreement with this previous value $\left(z_{\text {MOSFIRE }}=3.0768\right)$. All the remaining spectroscopic redshifts listed here are new
Table 1. SMUVS high-redshift $(z \geq 2)$ sources identified in the COSMOS/MUSE GTO field.

\begin{tabular}{|c|c|c|c|c|}
\hline Obj. ID & RA & Dec & $z_{\text {spec }}$ & QF \\
\hline 73023 & 150.1778406 & 2.1941108 & 3.8686 & 2 \\
\hline 73055 & 150.1044581 & 2.1943565 & 3.7710 & 9 \\
\hline 73162 & 150.1868076 & 2.1950781 & 4.4239 & 9 \\
\hline 73174 & 150.1807239 & 2.1949942 & 3.3375 & 3 \\
\hline 73452 & 150.0935903 & 2.1970951 & 3.0377 & 3 \\
\hline 73503 & 150.1657897 & 2.1971041 & 3.2774 & 9 \\
\hline $73761^{(1)}$ & 150.0973771 & 2.1988546 & 3.0747 & 3 \\
\hline 73993 & 150.1835347 & 2.2011801 & 3.2854 & 9 \\
\hline 74055 & 150.1698170 & 2.2012076 & 3.1317 & 9 \\
\hline 74237 & 150.1139483 & 2.2015449 & 3.4416 & 9 \\
\hline 74717 & 150.1050908 & 2.2068064 & 3.6090 & 9 \\
\hline 74990 & 150.0925832 & 2.2078347 & 3.5265 & 3 \\
\hline 75041 & 150.1815360 & 2.2085007 & 4.4443 & 3 \\
\hline 75190 & 150.1038713 & 2.2103898 & 3.2407 & 9 \\
\hline 75249 & 150.1209257 & 2.2102457 & 3.2212 & 9 \\
\hline 75267 & 150.1876116 & 2.2097678 & 2.4796 & 2 \\
\hline 75288 & 150.1393225 & 2.2109674 & 5.2944 & 3 \\
\hline 75461 & 150.1469868 & 2.2122848 & 3.4608 & 3 \\
\hline 75570 & 150.1181685 & 2.2128694 & 3.0031 & 9 \\
\hline 75825 & 150.1249549 & 2.2145933 & 5.9886 & 3 \\
\hline 76037 & 150.1601037 & 2.2163066 & 3.0060 & 3 \\
\hline 76038 & 150.0944809 & 2.2157858 & 3.3990 & 9 \\
\hline 76101 & 150.1703151 & 2.2160544 & 3.5259 & 3 \\
\hline 76321 & 150.1410657 & 2.2179212 & 3.9089 & 3 \\
\hline 76802 & 150.1064151 & 2.2215919 & 6.3044 & 9 \\
\hline 76829 & 150.1926599 & 2.2198279 & 3.0900 & 3 \\
\hline 76877 & 150.1659591 & 2.2216971 & 3.0913 & 3 \\
\hline 77599 & 150.1563335 & 2.2255982 & 3.1819 & 3 \\
\hline 77637 & 150.1334882 & 2.2276703 & 4.1650 & 3 \\
\hline 78106 & 150.1175785 & 2.2315227 & 2.9722 & 3 \\
\hline 78164 & 150.1058933 & 2.2303365 & 3.0097 & 3 \\
\hline 78359 & 150.1800388 & 2.2312780 & 2.1458 & 3 \\
\hline 78448 & 150.1866714 & 2.2319585 & 2.1723 & 2 \\
\hline 78588 & 150.1167228 & 2.2351184 & 3.8206 & 9 \\
\hline 78635 & 150.0881948 & 2.2344639 & 3.4881 & 3 \\
\hline 78718 & 150.1097010 & 2.2362429 & 3.7711 & 3 \\
\hline 90896 & 150.1160814 & 2.3273753 & 3.2644 & 9 \\
\hline 91354 & 150.1226590 & 2.3311551 & 3.0957 & 9 \\
\hline 91380 & 150.1274174 & 2.3309118 & 4.4672 & 9 \\
\hline
\end{tabular}

Notes. The SMUVS ID, position on the sky, measured spectroscopic redshift, and quality flags are listed. ${ }^{(1)} z_{\text {MOSFIRE }}=3.0768$ (Kriek et al. 2015).

(i.e. they are not present in the existing spectroscopic catalogs for the COSMOS field).

\subsection{MUSE spectra}

We focused our attention on the spectra of the 39 galaxies that have $z_{\text {spec }} \geq 2.0$. We separated the Ly $\alpha$ emitters from absorption line galaxies and AGNs, ending up with 36 sources at $z \gtrsim 3$ and 3 sources at redshift $2 \leq z \leq 3$, as described above.

\subsubsection{Lyman- $\alpha$ emitters}

We extracted the spectra of our Ly $\alpha$ emitters by identifying the extended area of the line emission. This area is defined by the pixels that have a signal-to-noise ratio higher than three. In order 
Table 2. Measured Ly $\alpha$ fluxes and luminosities for our SMUVS/MUSE sources.

\begin{tabular}{|c|c|c|c|c|}
\hline $\begin{array}{l}\text { SMUVS ID } \\
\text { no. }\end{array}$ & $\begin{array}{c}\text { Line center (Main) } \\
{[\AA]}\end{array}$ & $\begin{array}{c}\text { Line center (Sec.) } \\
{[\AA]}\end{array}$ & $\begin{array}{c}F_{\text {fit }} \\
{\left[10^{-18} \mathrm{erg} \mathrm{s}^{-1} \mathrm{~cm}^{-2}\right]}\end{array}$ & $\begin{array}{c}L_{\mathrm{fit}} \\
{\left[10^{42} \mathrm{erg} \mathrm{s}^{-1}\right]}\end{array}$ \\
\hline 73023 & $5917.9 \pm 0.6$ & $5920.4 \pm 1.6$ & $34.98 \pm 4.98$ & $5.18 \pm 0.74$ \\
\hline 73055 & $5799.2 \pm 0.5$ & - & $13.42 \pm 2.03$ & $1.87 \pm 0.28$ \\
\hline 73162 & $6594.1 \pm 0.2$ & - & $7.27 \pm 0.85$ & $1.48 \pm 0.17$ \\
\hline 73174 & $5272.9 \pm 0.2$ & - & $37.46 \pm 2.67$ & $3.89 \pm 0.28$ \\
\hline 73452 & $4909.5 \pm 0.2$ & - & $49.81 \pm 3.57$ & $4.13 \pm 0.30$ \\
\hline 73503 & $5201.2 \pm 0.6$ & $5192.7 \pm 0.8$ & $46.32 \pm 5.21$ & $4.61 \pm 0.52$ \\
\hline 73761 & $4958.2 \pm 0.2$ & $4950.0 \pm 0.2$ & $240.86 \pm 8.01$ & $20.56 \pm 0.68$ \\
\hline 73993 & $5210.7 \pm 0.6$ & - & $13.76 \pm 2.33$ & $1.38 \pm 0.23$ \\
\hline 74055 & $5024.1 \pm 0.6$ & - & $16.10 \pm 3.02$ & $1.44 \pm 0.27$ \\
\hline 74237 & $5399.2 \pm 0.4$ & $5405.9 \pm 0.6$ & $32.37 \pm 3.42$ & $3.62 \pm 0.38$ \\
\hline 74717 & $5604.2 \pm 0.4$ & - & $18.93 \pm 2.78$ & $2.37 \pm 0.35$ \\
\hline 74990 & $5508.9 \pm 0.2$ & - & $46.55 \pm 3.94$ & $5.52 \pm 0.47$ \\
\hline 75041 & $6618.6 \pm 0.3$ & $6610.5 \pm 0.8,6623.4 \pm 3.9$ & $17.11 \pm 3.32$ & $3.52 \pm 0.68$ \\
\hline 75190 & $5156.4 \pm 0.5$ & $5145.2 \pm 0.8$ & $37.35 \pm 5.16$ & $3.61 \pm 0.50$ \\
\hline 75249 & $5131.0 \pm 1.0$ & - & $17.94 \pm 3.65$ & $1.71 \pm 0.35$ \\
\hline 75288 & $7652.2 \pm 0.9$ & $7656.8 \pm 2.0$ & $22.52 \pm 4.55$ & $7.00 \pm 1.42$ \\
\hline 75461 & $5422.0 \pm 0.3$ & $5423.5 \pm 1.1$ & $21.55 \pm 3.29$ & $2.44 \pm 0.37$ \\
\hline 75570 & $4866.6 \pm 0.2$ & - & $68.61 \pm 4.62$ & $5.53 \pm 0.37$ \\
\hline 75825 & $8495.8 \pm 0.2$ & $8500.2 \pm 0.3$ & $43.01 \pm 3.23$ & $17.82 \pm 1.34$ \\
\hline 76037 & $4868.8 \pm 0.5$ & $4873.1 \pm 3.9$ & $26.42 \pm 10.52$ & $2.13 \pm 0.85$ \\
\hline 76038 & $5347.7 \pm 0.8$ & - & $16.98 \pm 2.96$ & $1.85 \pm 0.32$ \\
\hline 76101 & $5501.9 \pm 0.3$ & - & $11.91 \pm 1.58$ & $1.41 \pm 0.19$ \\
\hline 76321 & $5967.2 \pm 0.2$ & $5971.0 \pm 2.4$ & $50.53 \pm 8.81$ & $7.67 \pm 1.34$ \\
\hline 76802 & $8879.8 \pm 0.3$ & - & $24.76 \pm 3.13$ & $11.56 \pm 1.46$ \\
\hline 76829 & $4968.7 \pm 0.5$ & $4975.9 \pm 0.9$ & $32.19 \pm 4.05$ & $2.78 \pm 0.35$ \\
\hline 76877 & $4973.4 \pm 0.3$ & - & $35.59 \pm 3.44$ & $3.08 \pm 0.30$ \\
\hline 77599 & $5083.7 \pm 0.2$ & - & $64.82 \pm 6.18$ & $6.01 \pm 0.57$ \\
\hline 77637 & $6278.8 \pm 0.3$ & $6284.5 \pm 2.1$ & $26.72 \pm 3.46$ & $4.71 \pm 0.61$ \\
\hline 78106 & $4829.3 \pm 0.2$ & - & $48.94 \pm 4.58$ & $3.85 \pm 0.36$ \\
\hline 78164 & $4875.8 \pm 0.3$ & $4883.2 \pm 0.7$ & $59.26 \pm 6.23$ & $4.80 \pm 0.50$ \\
\hline 78588 & $5861.3 \pm 0.7$ & $5864.3 \pm 1.2$ & $39.44 \pm 8.04$ & $5.66 \pm 1.15$ \\
\hline 78635 & $5456.2 \pm 0.2$ & - & $70.35 \pm 4.68$ & $8.13 \pm 0.54$ \\
\hline 78718 & $5800.5 \pm 0.2$ & - & $121.65 \pm 7.48$ & $16.94 \pm 1.04$ \\
\hline 90896 & $5185.3 \pm 0.7$ & - & $24.07 \pm 3.85$ & $2.37 \pm 0.38$ \\
\hline 91354 & $4979.2 \pm 0.2$ & $4972.6 \pm 0.4$ & $40.92 \pm 3.37$ & $3.55 \pm 0.29$ \\
\hline 91380 & $6646.6 \pm 0.5$ & - & $38.46 \pm 4.09$ & $8.02 \pm 0.85$ \\
\hline
\end{tabular}

Notes. The ID, center of the main and the secondary peak, the measured flux from the fit, and the Ly $\alpha$ luminosity are reported.

to account for the instrument PSF, we also required a minimum area of 50 pixels. We then added the flux from the single pixels in the area of emission. Finally, we fitted one or two Gaussians to the obtained spectrum and measured the observed flux of the line.

Table 2 contains our line flux measurements, along with the luminosity of the Ly $\alpha$ line derived using the flux and the redshift measured for our objects. We measured the line flux by fitting our data with a number of Gaussians depending on the line profile shown in the spectrum. We then integrated the Gaussians in the wavelength interval containing the line to get the value of the flux reported in Table 2. The mean signal-to-noise ratio of our lines is $\sim 9.3$, with values spanning from 2.5 to 30 . The luminosities we measured are on the order of $10^{42}-10^{43} \mathrm{erg} \mathrm{s}^{-1}$, in line with the values published for MUSE-Wide data in other fields (Herenz et al. 2017, 2019).

Finally, we note that three different line profiles can be identified in our sample: a single-line profile, where the emission appears mostly symmetric; a blue bump profile, where in addi- tion to the main more intense line a secondary peak in the blue is visible; and a red tail profile, where the emission is either asymmetric with an extended tail in the red part of the spectrum or presents a secondary peak in the red. Figure 3 shows the zoomedin region of the spectra in the Ly $\alpha$ wavelength range. The plots in the figure also show that we cannot detect a continuum level in our data, thus we are not able to recognize P Cygni profiles, even if they are commonly observed for low-redshift objects. For the three profiles that we recognize, we report the following statistics. Out of 36 objects, 17 show a single-line profile $(\sim 47 \%), 7$ show a blue bump ( $19 \%), 11$ show a red tail $(\sim 31 \%)$, and 1 shows both a blue bump and a red tail $(\sim 3 \%)$.

The different profiles are caused by the condition and state of the medium in and around the galaxy. For example, a narrow single line can be caused by a reduced amount of scattering for the Ly $\alpha$ photons due to a low hydrogen column density. The blue bump can be caused either by re-emission by the medium of blueshifted Ly $\alpha$ photons or by a strong absorption at the resonant wavelength, leaving only the red tail and a fraction of the 

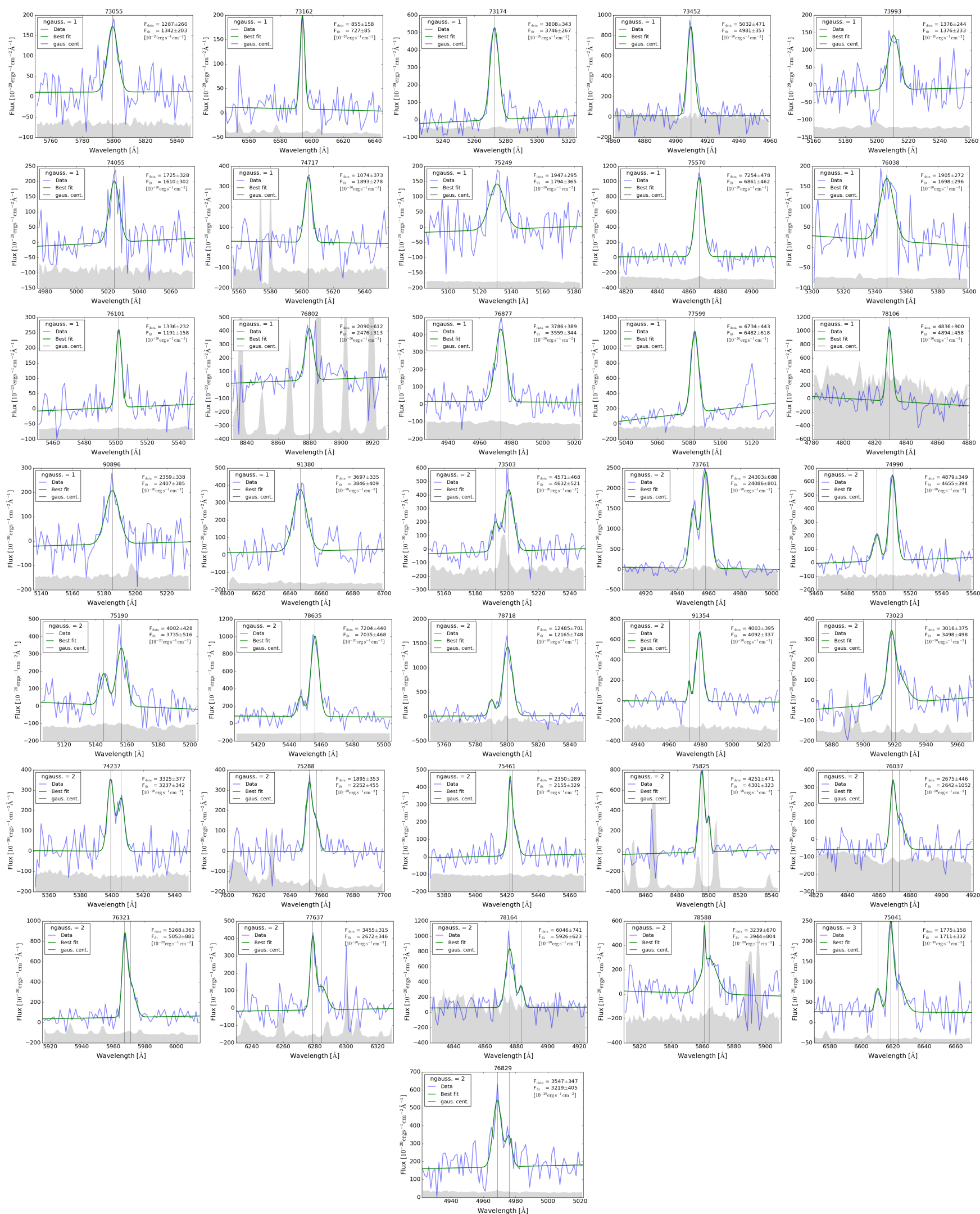

Fig. 3. Zoomed-in image of the spectra of our MUSE/SMUVS Ly $\alpha$ emitters. The wavelength range is chosen so that the Ly $\alpha$ line is visible and centered in the plot. The fit is performed by extracting the spectrum from the MUSE data cube in the region where the Ly $\alpha$ emission is $\sim 3 \sigma$ above the background or covers at least 50 pixels. The number of Gaussians used in the fit is determined after visual inspection of the shape of the spectrum and serves purely to measure the flux. All three line profiles are represented here. 
original emission. Finally, the red tail can give us information on the offset velocity of the medium, its optical depth, and the hydrogen column density. Radiative transfer models like the ones by Verhamme et al. (2008) or the more recent ones by Gronke (2017) can help disentangle the state of the gas and dust around the emitting galaxy. Applying such models is, however, outside of the scope of this paper, mainly due to the high noise in our spectra, and is left for follow-up studies.

\subsubsection{SMUVS/MUSE sources at $2 \leq z \leq 3$}

In addition to the 36 Ly $\alpha$ emitters, we identified 3 other highredshift objects in the range $2 \leq z \leq 3$. Object 75267 is an absorption line galaxy at redshift $z=2.4796$, classified as a $\mathrm{QF}=2$ spectrum. We report seeing the CIV doublet in absorption. Object 78359 is classified as a $\mathrm{QF}=3 \mathrm{AGN}$ at redshift $z=2.1458$ with broad-line emission of both [CIII] and CIV indicating the AGN activity. And finally, object 78448 is a galaxy at redshift $z=2.1723$ with $\mathrm{QF}=2$ emitting CIII and with possible FeII absorptions.

\subsection{Physical properties of the SMUVS MUSE galaxies inferred from broadband photometry}

We investigate the distribution of properties derived from SED fitting for our sample. Since the physical properties of each object are derived from SMUVS photometry by fitting its SED, we first need to check whether the redshift measured with MUSE matches the photometric redshifts originally derived by Deshmukh et al. (2018).

Out of the 691 SMUVS/MUSE sources for which we measured spectroscopic redshifts with high confidence, we find that 624 sources have a redshift compatible with the photometric value, while 62 are outliers. To define outliers we follow the same definition used by Deshmukh et al. (2018), i.e.,

$\sigma_{z}=\frac{\left|z_{\mathrm{spec}}-z_{\text {phot }}\right|}{\left(1+z_{\text {spec }}\right)}>0.15$,

where $z_{\text {spec }}$ is our spectroscopic redshift, while $z_{\text {phot }}$ is the photometric redshift of the SMUVS catalog.

Here we find a percentage of outliers of $\sim 10 \%$, which is somewhat larger than was found in Deshmukh et al. (2018) when comparing all their photometric redshifts with the available COSMOS spectroscopic redshifts, over the whole SMUVS/COSMOS area (the outlier fraction there was 5.5\%). This difference is perhaps not surprising, given that there is no source pre-selection in MUSE, while spectra taken with all other spectrographs are preferentially available for bright sources (for which the photometry has a higher signal-to-noise ratio, and thus the photometric analysis is more likely to yield good redshifts). In any case, it is reassuring that the percentage of redshift outliers that we obtain here is still reasonably low.

Before considering the SED fitting properties of our galaxies, we investigated the reason of the redshift discrepancies among the outliers. We focused on the $z \geq 2$ sources, which are the main interest here. Among the 39 high-redshift sources, we found 24 for which the photometric and spectroscopic redshifts are in good agreement. We analyzed the remaining 15 cases on an individual basis, in order to understand whether there is any problem in the spectroscopic and/or photometric analysis, or whether the SMUVS/MUSE sources matching is correct. For eight of the outliers we found that the photometry is contaminated by a brighter neighbor; for the other seven we found no apparent photometric contamination. The redshift discrepancy is produced by
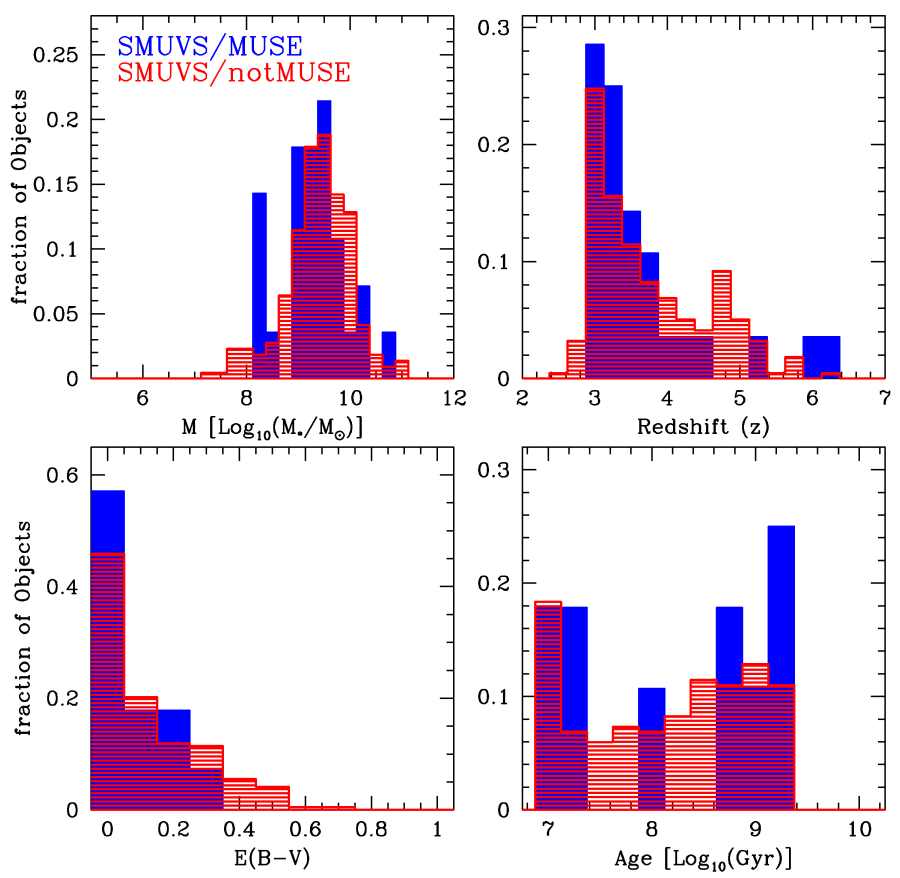

Fig. 4. Histogram of the three main properties (age, extinction, and stellar mass) derived from the SMUVS photometry and the redshifts measured by MUSE. We compare our sample of 28 Ly $\alpha$ emitters to the SMUVS sample of complementary high-z objects (218 galaxies).

either the galaxy being fainter than the limiting magnitude of the survey (four cases) or the SMUVS detection actually being two unresolved, separate sources (three cases). In the first situation the photometry of the source is likely unreliable in some bands and in the second case both sources influence the values measured in the photometry, but only one of them emits $\operatorname{Ly} \alpha$ and is detected in MUSE. In both cases the photometric fit results in lower $z_{\text {phot }}$ in comparison to the $z_{\text {spec }}$.

As the next step, we redid the SED fitting of our high-redshift galaxies with uncontaminated photometry, fixing their redshifts to the MUSE-based spectroscopic value. We used the code LePhare $^{1}$ (Arnouts et al. 1999; Ilbert et al. 2006), with the same template family and parameter values as in Deshmukh et al. (2018), and considered the same SMUVS 28-band input catalog. We then reran LePhare with fixed redshifts also on the sources that did not show a severe contamination, and found that six of them could be recovered. We thus obtained a final sample of 30 sources (instead of the previous 24) with physical properties derived from photometry, 28 of which are MUSE Ly $\alpha$ emitters.

We see that the SMUVS/MUSE sources have extinction values between $0.0 \leq E(B-V) \leq 0.3$, with $\sim 70 \%$ having $E(B-V) \leq$ 0.1 , as we would expect from systems that show prominent Ly $\alpha$ lines. Nonetheless, there are still sources that have higher extinctions $(0.2 \leq E(B-V) \leq 0.3)$, hinting at an even higher unattenuated Ly $\alpha$ luminosity in those cases. The stellar mass range covered by our sources is from $\sim 1.5 \times 10^{8} M_{\odot}$ to $\sim 7 \times 10^{11} M_{\odot}$, with a mean value of $\sim 10^{9} M_{\odot}$. Finally, we see that our objects have ages ranging from $10 \mathrm{Myr}$ to $2 \mathrm{Gyr}$.

In the next subsection we compare the distribution of the derived SED properties for the Ly $\alpha$ emitters and other SMUVS sources with $z \geq 2.9$ (see also Fig. 4).

1 http://www.cfht.hawaii.edu/ arnouts/lephare.html 
Table 3. Results of all the KS tests performed in this work.

\begin{tabular}{|c|c|c|c|}
\hline \multirow{3}{*}{$\begin{array}{l}\text { Test } \\
\text { performed on }\end{array}$} & \multicolumn{3}{|c|}{$p$-values for } \\
\hline & \multicolumn{2}{|c|}{$\begin{array}{c}\text { SMUVS/... } \\
\text { MUSE vs. notMUSE }\end{array}$} & \multirow{2}{*}{$\begin{array}{c}\text { SMUVS/MUSE } \\
\text { vs. } \\
\text { MUSE/NS }\end{array}$} \\
\hline & original & mass matched & \\
\hline Age & 0.43 & $3.5 \times 10^{-2}$ & - \\
\hline Extinction & 0.61 & $2.3 \times 10^{-6}$ & _- \\
\hline Stellar mass & 0.08 & 0.997 & - \\
\hline Redshift & 0.12 & - & 0.02 \\
\hline$L_{\mathrm{Ly} \alpha}$ & - & - & $1.5 \times 10^{-3}$ \\
\hline$F_{\mathrm{Ly} \alpha}$ & - & - & $4 \times 10^{-6}$ \\
\hline
\end{tabular}

\subsection{Comparison of SED properties for SMUVS galaxies with and without MUSE identification}

We compare the derived SED properties of the SMUVS $z>$ 2.9 sources identified with MUSE (i.e., those with a spectroscopic redshift measurement with $\mathrm{QF} \geq 2$ ) with those of the SMUVS $z \gtrsim 2.9$ galaxies that are not MUSE-detected, in the same field. Our aim is to investigate whether there are significant differences in these properties, in particular to understand whether the most prominent Ly $\alpha$ emitters at $z>3$ are characterized by special values in their physical properties (stellar mass, star formation histories, dust extinction, etc.).

Prior to comparing the SED properties of our objects, we tested whether SMUVS/MUSE and SMUVS/notMUSE galaxies come from the same parent absolute-magnitude distribution. The sample of galaxies we consider is 218 SMUVS galaxies with redshift $2.9 \lesssim z_{\text {phot }} \lesssim 7$ and the 28 MUSE Ly $\alpha$ emitters for which our spectroscopic redshifts and the SMUVS photometric redshift estimates are in good agreement (as defined in Sect. 3.3). The comparison is performed on all 28 photometric bands available to SMUVS separately. We performed a Kolmogorov-Smirnov (KS) test on the statistics of the absolute magnitudes derived with LePhare for our two samples. The results of these tests are that the D parameter assumes values $\lesssim 0.25$ over all the bands tested, and the $p$-value is always $\geq 0.08$. This indicates that our SMUVS high-redshift MUSE-detected and non-detected sources show no statistically significant difference.

To test whether the sample size can influence the outcome of the test, we reduced the SMUVS sample randomly 10000 times to subsamples containing an average of 40 objects. The KS test is then performed on these smaller samples and the results are compared to the test on the whole SMUVS sample by means of their statistics. We conclude that sample size does not matter statistically in our case.

Figure 4 shows the distribution of the physical properties of our two samples derived from the SED fit. As can be expected, the redshift distribution of both samples is biased towards lower redshifts in the range $2.9 \leq z<6.6$ covered by MUSE. The distribution of the stellar mass for our SMUVS/MUSE sample is between $8<\log _{10}\left(M_{*}\right)<11$, with a peak at $\sim 10^{9.5} M_{*}$. This sample is also mostly composed of galaxies with little dust content. Nevertheless, some of our objects have $E(B-V)>0.25$ and are still visible as Ly $\alpha$ emitters. Unless the emission is seen through a gap in the dust distribution, this would make them extremely bright in $\operatorname{Ly} \alpha$ to overcome such higher values of dust extinction and still be visible. Finally, the age distribution exhibits a bimodality, either classifying our galaxies as very young ( $\$ 100 \mathrm{Myr}$ ) or $\sim 1$ Gyr old. We comment further on this feature in the next subsection. Figure 4 confirms our previous
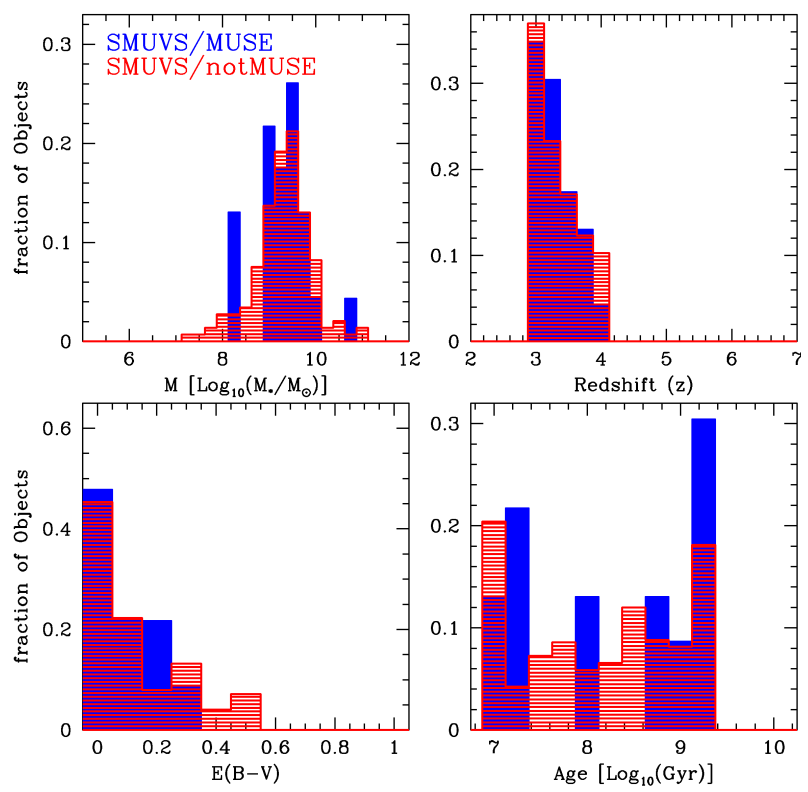

Fig. 5. Redshift cut and new stellar mass distribution due to the cut as they were before the mass matching in the upper panels. The mass matched histograms of the extinction $E(B-V)$ and the age derived from the SMUVS photometry for 23 SMUVS/MUSE and 146 SMUVS/notMUSE sources in the redshift range $3 \leq z \leq 4$ are shown in the lower panels.

analysis on the input photometric bands used in SMUVS: The distribution of the properties of the SMUVS/MUSE and SMUVS/notMUSE sample are not statistically different.

After we performed a KS test on the physical properties, we concluded that, compared to the SMUVS/notMUSE sample, the spectroscopically detected galaxies generally have lower dust extinction, about the same mass and age distributions, and fewer objects are detected in higher redshift regimes $(4 \leq z \leq 6.6)$, but we see no significant statistical difference in any of the properties. The individual results of our KS tests can be seen in Table 3.

To further test the distribution of the physical properties in both samples, we constructed Fig. 5. We restricted ourselves to analyzing galaxies in the redshift range $\sim(3,4)$, to limit the effect of galaxy evolution with redshift and because most of the SMUVS/MUSE objects lie in that range. We then also stellar mass matched the SMUVS/notMUSE galaxies to SMUVS/MUSE. The redshift cut applies to all the panels, while the mass matching is shown only for the extinction and the ages. We can see how the age distribution appears very similar in the two samples and how the distribution of redshift and masses do not deviate much from what we saw in Fig. 4, before the redshift cut was applied. The interesting panel is the one showing the extinction. We can see now how applying a cut in redshift and stellar mass matching the SMUVS/notMUSE sample reveals a slight difference in the distribution. SMUVS/MUSE shows preferentially lower extinctions compared to SMUVS/notMUSE, which deviates from $E(B-V)>$ 0.2 onward and drives the difference. Again, the results of a formal KS test can be seen in Table 3.

\subsection{Further study of the age bimodality}

We study the implications of an age bimodality in our MUSEdetected SMUVS sample by comparing the stellar mass-age and $E(B-V)$-age relations of SMUVS/MUSE to the relations found in SMUVS/notMUSE (see Fig. 6). We can see, as is expected from the current view on galaxy evolution, that the younger 

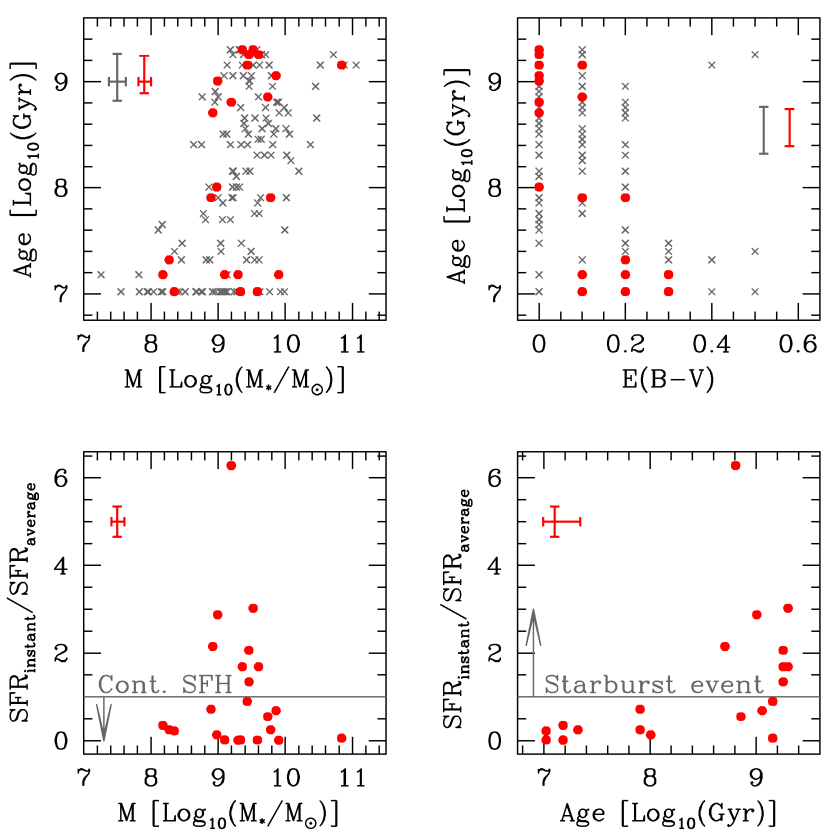

Fig. 6. Upper panels: mass-age relation on the left and $E(B-V)-$ age relation on the right for both our SMUVS detected samples. Here, SMUVS/MUSE is represented in red, while SMUVS/notMUSE is plotted in gray. We see that the older objects are generally more massive and that the younger objects experience generally more extinction than older objects. Lower panels: test of the lower limit of the instantaneous SFR derived from our Ly $\alpha$ flux measurements against an average star formation rate obtained by dividing the stellar mass by the age of the galaxy obtained from the SED fit. We see on the left that the higher SFR is associated with intermediate-mass objects, and on the right that older objects have a higher SFR than what would be expected if they had continuously formed stars in one single episode. The gray line in the plots indicates where the ratio between SFRs is unity. The error bars shown in the plots are representative of the average errors on the galactic properties.

objects tend to be less massive than the older objects and that they are in general more obscured than older objects. We associate these characteristics with the fact that the younger galaxies in our sample are currently experiencing their first star formation event. The older objects in contrast have already formed the bulk of their stars, and thus show older populations and have less dust in their surroundings.

We notice however that all SMUVS/MUSE objects are detected in Ly $\alpha$ emission and so we expect them to be actively star-forming. We argue that the star formation that we see in the older objects is not part of the first event, but a separated second episode of star formation. To test this, we generated the lower plots in Fig. 6. We compare the SFR obtained if we assume a continuous star formation throughout the lifetime of our objects and the lower limit of the SFR given by the Ly $\alpha$ emission we detect. The first value is obtained by dividing the stellar mass given by the SED fit with the age of the galaxy and represents the average of the SFR. The second value is obtained by applying the conversion $L_{\mathrm{H} \alpha}=L_{\mathrm{Ly} \alpha} * \frac{1}{8.7}$, which gives us a lower limit for the luminosity in the $\mathrm{H} \alpha$ line and for which we chose to use the $\mathrm{Ly} \alpha-\mathrm{H} \alpha$ conversion factor by $\mathrm{Hu}$ et al. (1998). We then use the Kennicutt (1998) equation to obtain the SFR from the $\mathrm{H} \alpha$ luminosity to obtain the lower limit of the instantaneous SFR.

By comparing these two values we can qualitatively say whether a galaxy is consistent with a monotonically declining star formation history $\left(\mathrm{SFR}_{\text {instant }} / \mathrm{SFR}_{\text {average }} \leq 1\right)$ or if a second star formation episode is needed to explain the SFR

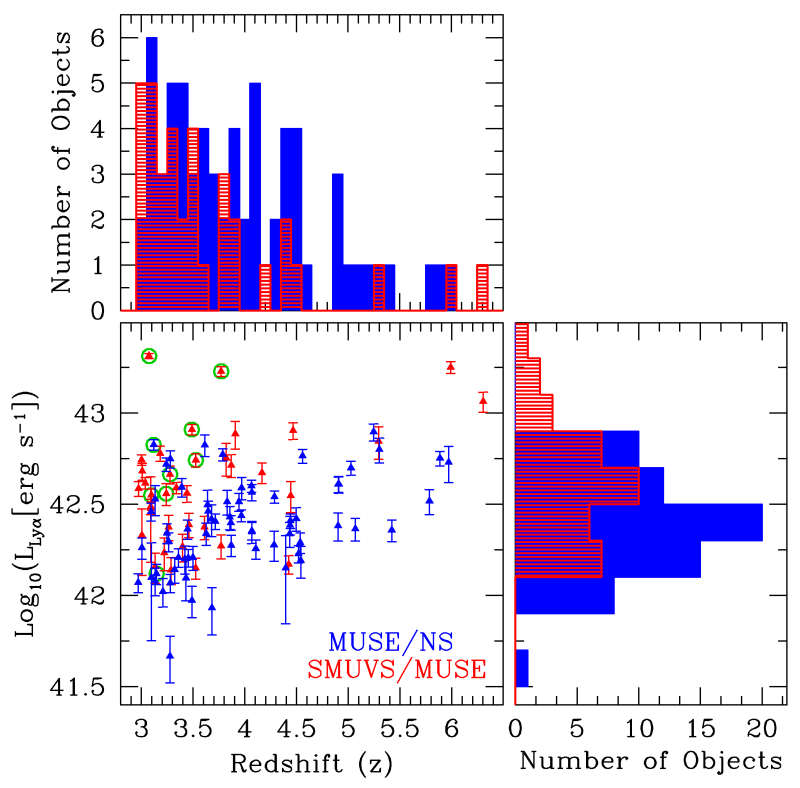

Fig. 7. Upper panel: comparison in redshift distribution between the SMUVS sources (red) and the sources identified in the Blind search (blue). Left panel: Ly $\alpha$ luminosity plotted as a function of redshift for our sources. The green circles identify the blue bump objects. Right panel: histogram of the measured luminosities for both our samples. The blind search objects show slightly fainter luminosities compared to the SMUVS sources.

$\left(\mathrm{SFR}_{\text {instant }} / \mathrm{SFR}_{\text {average }}>1\right)$. We see that galaxies that are experiencing a second event are intermediate-mass objects $(9 \lesssim$ $\left.\log _{10}\left(M_{*}\right) \leq 10\right)$ and are exclusively old. We argue that the main component of the stellar population has formed in early times with the first star formation event and is what the SMUVS photometry detects, while the new stars being formed are detected by MUSE. We conclude that these objects are probably experiencing a rejuvenation event, and that their star formation restarted after the stellar bulk was formed, about $1 \mathrm{Gyr}$ before the time we observe them.

\subsection{Additional MUSE high-redshift sources not present in SMUVS}

Additionally to the Ly $\alpha$ emitters in the SMUVS/MUSE sample, we also performed a blind search in the MUSE cubes and found 66 other sources presenting a secure or possible Ly $\alpha$ emission $(\mathrm{QF}=2,3$, and 9). These new sources (hereafter MUSE/NS) have been identified by visually inspecting the MUSE data cubes, and do not have a previous spectroscopic confirmation. We also verified that, in the specific framework of our analysis, performing an automated search in our cubes (i.e., by using the software LSDCat, Herenz \& Wisotzki 2017) rather than a visual one would not add new sources to our secure detections $(\mathrm{QF}>1)$. Since the MUSE/NS sample is not present in the SMUVS cata$\log$, we have information on the redshift and measurements of the Ly $\alpha$ flux and luminosity, but no estimate of the physical properties of these objects from SED fitting. The MUSE/NS sources and all their related quantities are listed in Table B.1.

To test whether these objects belong to a different population from the galaxies selected in SMUVS, we verified that none of these new sources was situated in masked areas of the survey. In fact, 61 of them were never selected for the catalog to begin with and 5 have a SMUVS neighbor within 1", but are clearly different objects. Since the SMUVS galaxies were detected based on a 
Table 4. Position and redshift of the three secure associations identified via our method.

\begin{tabular}{lcccc}
\hline \hline $\begin{array}{l}\text { Association } \\
\text { number }\end{array}$ & Obj-ID & RA & Dec & $z$ \\
\hline A1 & NS67 & 150.1634469 & 2.210140164 & 3.8656 \\
& NS92 & 150.1805399 & 2.235132627 & 3.8673 \\
\hline A2 & 76829 & 150.1926599 & 2.2198279 & 3.09 \\
& 76877 & 150.1659591 & 2.2216971 & 3.0913 \\
\hline A3 & NS4 & 150.0902794 & 2.208985301 & 4.0681 \\
& NS75 & 150.094679 & 2.230024846 & 4.0667 \\
\hline
\end{tabular}

prior selection in the UltraVISTA $H K_{s}$ stacks, we conclude that these MUSE/NS sources are faint in the $H K_{s}$ stacks and/or in the images from the Spitzer $3.6 \mu \mathrm{m}$ and $4.5 \mu \mathrm{m}$ bands.

Given that the UltraVISTA images are deep, we can safely assume that the reason these objects are undetected is that they are below the mass limit of the survey. Even without having performed an SED fit, we can state that the MUSE/NS sample will likely have a very different mass distribution compared to the SMUVS/MUSE sample. This is likely the source of the discrepancies we find between the two populations. We put an upper limit on their stellar mass by citing the $50 \%$ completeness limit reported in Table 1 of Deshmukh et al. (2018). For galaxies in $3.0 \leq z \leq 4.0$ the upper limit mass is $\log _{10}\left(M_{*} / M_{\odot}\right)=9.0$, for the range $4.0<z \leq 5.0$ it is 9.2 , and finally for galaxies with $5.0<z \leq 6.0$ it is 9.4 .

We measured the Ly $\alpha$ emission and luminosity for the MUSE/NS sample in the same way as we did for the SMUVS/MUSE sources. The first difference we noticed is that the number of blue bump profiles is strongly reduced compared to the SMUVS/MUSE sample (2/66 against $7 / 36$, or $\sim 3 \%$ against $\sim 19 \%$ ). Furthermore, the single-line profile appears in 44 spectra, constituting $\sim 67 \%$ of the sample, which makes it more prominent than in the SMUVS/MUSE sample, where it was observed $\sim 47 \%$ of the time. Finally, the red tail profile appears in 20 of our galaxies and is about as frequent as in the SMUVS/MUSE sample (MUSE/NS 30\%, SMUVS/MUSE $\sim 31 \%$ ). If we compare SMUVS/MUSE to MUSE/NS, both in line flux and luminosity using a KS test, we see that their distributions are different and that MUSE/NS are the fainter objects. This confirms the fact that blue bump profiles are harder to detect, and thus the single-line profiles increase in number for the fainter sources. Further evidence for this interpretation is that the blue bump objects are found in the brighter objects located at $3 \leq z<4$ of the SMUVS/MUSE (green circles in Fig. 7). The results of these KS tests can again be found in Table 3 .

The right panel of Fig. 7 illustrates this trend by showing the distribution of $L_{\mathrm{Ly} \alpha}$ for the two samples. We also plotted their distribution in redshift and how their luminosity evolves with it, in the upper and left panel of Fig. 7, respectively. We see in the central panel of Fig. 7 that for both samples the measured Ly $\alpha$ luminosity plotted against redshift increases with it, as can be expected given the detection limit on the line flux. More interestingly, the MUSE/NS sources seem to lie close enough in redshift space to the SMUVS/MUSE sources, suggesting they could belong to the same physical structure. To test if this is the case, we defined a $\Delta z$ dependent on redshift, such that two objects with said distance in redshift lie $2 \mathrm{Mpc}$ apart. We then expanded our check also to the position on the sky and defined a sphere of $2 \mathrm{Mpc}$ radius as our criterion to check for associations.

We find that we can identify nine associations in the area covered by MUSE. If we restrict ourselves to only considering
$\mathrm{QF}=3,4$ objects, then the number of associations we find drops to three (see Table 4). We compared the number of associations we find with MUSE in our small area to the number of associations we could find in the part of the COSMOS field covered by SMUVS using spectroscopic confirmed objects currently known in the literature. We chose spectroscopic sources with $\mathrm{QF}=3,4$ only and applied the same criteria used on the MUSE data to identify associations. We find 16 associations over the entirety of the SMUVS/COSMOS field. If we assume that the MUSE rate in our small area is indicative of what we could find if MUSE covered SMUVS/COSMOS entirely, then we would expect to find $\sim 360$ associations in this larger area. Unless cosmic variance is playing a big role in the area MUSE covered in our data, we estimate that MUSE has a $\sim 20 \times$ higher chance of detecting objects that could be physically linked, confirming the usefulness of MUSE for unbiased source detection.

\section{Summary and conclusions}

We made use of publicly available MUSE data in the COSMOS field to analyze a sample of 2997 photometrically selected galaxies from the SMUVS survey over an area of $20.79 \mathrm{arcmin}^{2}$. We managed to detect and measure the redshift of 691 objects, of which 39 are located at $z \geq 2$. For these sources, we report two absorption line galaxies and one AGN in addition to 36 Lyman- $\alpha$ emitters. Of these 39 sources, all but 1 are new redshift measurements not previously present in the literature. Furthermore, we identify 66 additional Ly $\alpha$ emitters by performing a blind search in the MUSE cubes. The values we measured for the $\operatorname{Ly} \alpha$ flux and luminosity of our sources are in line with other works using MUSE pointings with similar depth and conditions (Herenz et al. 2017, 2019). We also detect three different line profiles in our combined Ly $\alpha$ sample, hinting at different medium conditions in and around our objects. A quantitative analysis of these features was, however, beyond the scope of this paper, whose goal was instead to investigate the differences in physical properties between the sources identified in the SMUVS catalog also detected in MUSE and those that could not be detected in MUSE.

The main result of our paper is to compare the physical properties of the SMUVS/MUSE and SMUVS/notMUSE sources. While there are some differences in the distribution of $E(B-V)$, stellar mass, and age in the two samples, we find that their overall distribution does not vary substantially. We know that the SMUVS catalog is more sensitive to the brighter, more massive galaxies. MUSE instead has the only bias of being able to select objects that are at least bright enough in line emission to be detected. For $z>2.9$ galaxies, this means they are bright Ly $\alpha$ emitters, intense star-forming objects with little dust attenuation/HI absorption. Not finding a significant difference in physical properties between our two samples, even after applying a redshift cut and mass matching them, could imply that the SMUVS selected Ly $\alpha$ emitters we observe in MUSE are similar to the general population in SMUVS. Although SMUVS is not the largest nor deepest survey in COSMOS, it is the Spitzer survey with the largest area for its depth (only shallower than CANDELS, which covers an area 12 times smaller in COSMOS). We can thus probe a sample of galaxies that is more complete in parameter space than ever before.

After mass matching our samples, we see that SMUVS/ MUSE is less obscured than SMUVS/notMUSE. We also note how the age distribution of our Ly $\alpha$ emitters shows a bimodality. Both these results have been found in other studies where Ly $\alpha$ information has been combined with photometric SED fit- 
ting (Lai et al. 2008; Finkelstein et al. 2009; Pentericci et al. 2009). Furthermore, we deepened our study of the bimodality in the age of our sample by studying the SFR of our objects qualitatively. We find that the younger objects are most likely experiencing their first burst of star formation. The older objects in our sample show instead a lower limit to their instantaneous SFR, derived from their Ly $\alpha$ luminosity, that suggests they are undergoing a second burst of star formation, while the light of the galaxy is dominated by an older population of stars. This rejuvenation effect has also recently been observed in low-redshift objects by Angthopo et al. (2019) and Cooke et al. (2019).

We also compared the SMUVS/MUSE and MUSE/NS samples. Using MUSE data allowed us an unbiased detection of all the sources that are bright in Ly $\alpha$. We were able to perform a blind search in the MUSE cubes and enlarge our sample of Ly $\alpha$ emitters by a factor of $\sim 3$. MUSE thus confirms its potential for systematic searches of a given area in the sky. This sensitivity to emission lines also allowed us to detect three secure and previously undiscovered physical galaxy associations.

What is puzzling, however, is that MUSE detects MUSE/NS objects at luminosities around $10^{42} \mathrm{erg} \mathrm{s}^{-1}$ and below, but does not detect such lower luminosities for the SMUVS/MUSE sample (see right histogram in Fig. 7). We determined that the MUSE/NS sample lies most likely below the mass completeness limit of the SMUVS survey. We note, however, that the Ly $\alpha$ luminosities of the MUSE/NS sample have a significant overlap with the SMUVS/MUSE luminosities. Since the Ly $\alpha$ line intensity is linked to the ionizing radiation emitted and not the mass of the object, this overlap is not surprising. It does, however, point out that we cannot attribute the lack of lower luminosity SMUVS/MUSE objects to a simple scaling effect due to the mass-selection in SMUVS. At present we do not have a definitive solution for this issue.

The area of improvement in this study is the depth of the pointings. Longer exposure times could yield a higher detection rate for the SMUVS selected sources (to date about 2/3 could not be detected) and deep enough spectra to measure a continuum. This would allow us to more accurately investigate the line shapes (we could distinguish P Cygni profiles from single lines) and also measure equivalent widths for the brighter objects.

Acknowledgements. The authors would like to thank the anonymous referee for the helpful and constructing feedback. GR, GBC, KIC and SD acknowledge funding from the European Research Council through the award of the Consolidator Grant ID 681627-BUILDUP. Based on observations collected at the European Southern Observatory under ESO Programme IDs 095.A0240(A), 096.A-0090(A), 097.A-0160(A), 098.A-0017(A). Also based in part on observations carried out with the Spitzer Space Telescope, which is operated by the Jet Propulsion Laboratory, California Institute of Technology under a contract with NASA. This research made use of Astropy, (http://www . astropy.org) a community-developed core Python package for Astronomy (Astropy Collaboration 2013, 2018).

\section{References}

Acquaviva, V., Vargas, C., Gawiser, E., \& Guaita, L. 2012, ApJ, 751, L26 Angthopo, J., Ferreras, I., \& Silk, J. 2019, MNRAS, 488, L99 Arnouts, S., Cristiani, S., Moscardini, L., et al. 1999, MNRAS, 310, 540 Ashby, M. L. N., Caputi, K. I., Cowley, W., et al. 2018, ApJS, 237, 39 Astropy Collaboration (Robitaille, T. P., et al.) 2013, A\&A, 558, A33 Astropy Collaboration (Price-Whelan, A. M., et al.) 2018, AJ, 156, 123

Bacon, R., Accardo, M., Adjali, L., et al. 2010, in Ground-based and Airborne Instrumentation for Astronomy III, Proc. SPIE, 7735, 773508

Bacon, R., Piqueras, L., Conseil, S., Richard, J., \& Shepherd, M. 2016, Astrophysics Source Code Library [record ascl:1611.003]

Bertin, E., \& Arnouts, S. 1996, A\&AS, 117, 393
Bridge, J. S., Hayes, M., Melinder, J., et al. 2018, ApJ, 852, 9 Caputi, K. I., Cirasuolo, M., Dunlop, J. S., et al. 2011, MNRAS, 413, 162 Caputi, K. I., Deshmukh, S., Ashby, M. L. N., et al. 2017, ApJ, 849, 45 Cooke, K. C., Kartaltepe, J. S., Tyler, K. D., et al. 2019, ApJ, 881, 150 Cowley, W. I., Caputi, K. I., Deshmukh, S., et al. 2018, ApJ, 853, 69 Cowley, W. I., Caputi, K. I., Deshmukh, S., et al. 2019, ApJ, 874, 114 Curtis-Lake, E., McLure, R. J., Dunlop, J. S., et al. 2013, MNRAS, 429, 302 De Barros, S., Pentericci, L., Vanzella, E., et al. 2017, A\&A, 608, A123 Deshmukh, S., Caputi, K. I., Ashby, M. L. N., et al. 2018, ApJ, 864, 166 Diener, C., Wisotzki, L., Schmidt, K. B., et al. 2017, MNRAS, 471, 3186 Dijkstra, M. 2017, ArXiv e-prints [arXiv:1704 . 03416]

Erb, D. K., Steidel, C. C., \& Chen, Y. 2018, ApJ, 862, L10

Fazio, G. G., Hora, J. L., Allen, L. E., et al. 2004, ApJS, 154, 10

Finkelstein, S. L., Rhoads, J. E., Malhotra, S., \& Grogin, N. 2009, ApJ, 691, 465 Grogin, N. A., Kocevski, D. D., Faber, S. M., et al. 2011, ApJS, 197, 35 Gronke, M. 2017, A\&A, 608, A139

Guaita, L., Acquaviva, V., Padilla, N., et al. 2011, ApJ, 733, 114 Gurung-Lopez, S., Orsi, A. A., \& Bonoli, S. 2019, MNRAS, 490, 733 Hao, C.-N., Huang, J.-S., Xia, X., et al. 2018, ApJ, 864, 145 Herenz, E. C., \& Wisotzki, L. 2017, A\&A, 602, A111

Herenz, E. C., Urrutia, T., Wisotzki, L., et al. 2017, A\&A, 606, A12 Herenz, E. C., Wisotzki, L., Saust, R., et al. 2019, A\&A, 621, A107 Hernán-Caballero, A., Pérez-González, P. G., Diego, J. M., et al. 2017, ApJ, 849, 82

Hu, E. M., Cowie, L. L., \& McMahon, R. G. 1998, ApJ, 502, L99 Ilbert, O., Arnouts, S., McCracken, H. J., et al. 2006, A\&A, 457, 841 Kakiichi, K., \& Gronke, M. 2019, ApJ, submitted [arXiv:1905. 02480] Karman, W., Caputi, K. I., Trager, S. C., Almaini, O., \& Cirasuolo, M. 2014, A\&A, 565, A5

Karman, W., Caputi, K. I., Caminha, G. B., et al. 2017, A\&A, 599, A28 Kennicutt, Jr., R. C. 1998, ARA\&A, 36, 189

Kimm, T., Blaizot, J., Garel, T., et al. 2019, MNRAS, 486, 2215

Koekemoer, A. M., Faber, S. M., Ferguson, H. C., et al. 2011, ApJS, 197, 36 Kovač, K., Lilly, S. J., Cucciati, O., et al. 2010, ApJ, 708, 505 Kriek, M., Shapley, A. E., Reddy, N. A., et al. 2015, ApJS, 218, 15 Lai, K., Huang, J.-S., Fazio, G., et al. 2008, ApJ, 674, 70 Le Fèvre, O., Vettolani, G., Garilli, B., et al. 2005, A\&A, 439, 845 Lilly, S. J., Le Fèvre, O., Renzini, A., et al. 2007, ApJS, 172, 70 Mallery, R. P., Mobasher, B., Capak, P., et al. 2012, ApJ, 760, 128 Marchi, F., Pentericci, L., Guaita, L., et al. 2019, A\&A, 631, A19 Martin, C. L., Dijkstra, M., Henry, A., et al. 2015, ApJ, 803, 6 Martinache, C., Rettura, A., Dole, H., et al. 2018, A\&A, 620, A198 Mas-Hesse, J. M., Kunth, D., Tenorio-Tagle, G., et al. 2003, ApJ, 598, 858 McCracken, H. J., Milvang-Jensen, B., Dunlop, J., et al. 2012, A\&A, 544, A156 McLinden, E. M., Rhoads, J. E., Malhotra, S., et al. 2014, MNRAS, 439, 446 Nakajima, K., Fletcher, T., Ellis, R. S., Robertson, B. E., \& Iwata, I. 2018, MNRAS, 477, 2098

Ono, Y., Ouchi, M., Shimasaku, K., et al. 2010, MNRAS, 402, 1580 Orlitová, I., Verhamme, A., Henry, A., et al. 2018, A\&A, 616, A60 Pentericci, L., Grazian, A., Fontana, A., et al. 2009, A\&A, 494, 553 Pentericci, L., Grazian, A., Scarlata, C., et al. 2010, A\&A, 514, A64

Piqueras, L., Conseil, S., Shepherd, M., et al. 2017, ArXiv e-prints [arXiv:1710.03554]

Remolina-Gutiérrez, M. C., \& Forero-Romero, J. E. 2019, MNRAS, 482, 4553 Scoville, N., Aussel, H., Brusa, M., et al. 2007, ApJS, 172, 1

Shapley, A. E., Steidel, C. C., Pettini, M., \& Adelberger, K. L. 2003, ApJ, 588, 65

Smith, A., Ma, X., Bromm, V., et al. 2019, MNRAS, 484, 39

Sobral, D., Matthee, J., Darvish, B., et al. 2018, MNRAS, 477, 2817

Soto, K. T., Lilly, S. J., Bacon, R., Richard, J., \& Conseil, S. 2016, MNRAS, 458, 3210

Taniguchi, Y., Scoville, N., Murayama, T., et al. 2007, ApJS, 172, 9

Tenorio-Tagle, G., Silich, S. A., Kunth, D., Terlevich, E., \& Terlevich, R. 1999, MNRAS, 309, 332

Urrutia, T., Wisotzki, L., Kerutt, J., et al. 2019, A\&A, 624, A141

Vanzella, E., Nonino, M., Cupani, G., et al. 2018, MNRAS, 476, L15

Verhamme, A., Schaerer, D., Atek, H., \& Tapken, C. 2008, A\&A, 491, 89

Verhamme, A., Garel, T., Ventou, E., et al. 2018, MNRAS, 478, L60

Weilbacher, P. M., Roth, M. M., Pécontal-Rousset, A., \& Bacon, R. 2006, New Astron. Rev., 50, 405

Weilbacher, P. M., Streicher, O., Urrutia, T., et al. 2012, in Software and Cyberinfrastructure for Astronomy II, Proc. SPIE, 8451, 84510B

Weilbacher, P. M., Streicher, O., Urrutia, T., et al. 2014, in Astronomical Data Analysis Software and Systems XXIII, eds. N. Manset, \& P. Forshay, ASP Conf. Ser., 485, 451

Werner, M. W., Roellig, T. L., Low, F. J., et al. 2004, ApJS, 154, 1

Yuma, S., Ohta, K., Yabe, K., et al. 2010, ApJ, 720, 1016

Zhang, H., Ouchi, M., Itoh, R., et al. 2019, ApJ, submitted [arXiv:1905 . 09841] 


\section{Appendix A: Complete redshift catalog}

Table A.1 shows the first five rows of our complete redshift cata$\log$. We only include all redshift measurements that have $\mathrm{QF}>1$ in this table.
Table A.1. First five rows of the full redshift catalog for the $20.79 \operatorname{arcmin}^{2}$ covered by MUSE.

\begin{tabular}{lcccc}
\hline \hline Obj. ID & RA & Dec & $z_{\text {spec }}$ & QF \\
\hline 72926 & 150.1057453 & 2.1931866 & 0.9231 & 9 \\
73005 & 150.1425919 & 2.1927638 & 0.9930 & 2 \\
73023 & 150.1778406 & 2.1941108 & 3.8686 & 2 \\
73053 & 150.0958292 & 2.1924913 & 0.7581 & 3 \\
73055 & 150.1044581 & 2.1943565 & 3.7710 & 9 \\
$\vdots$ & $\vdots$ & $\vdots$ & $\vdots$ & $\vdots$ \\
\hline
\end{tabular}

Notes. The columns list the ID, position on the sky, measured redshift, and quality flag, respectively. All the entries are secure redshift measurements $(\mathrm{QF}>1)$. The full table is available at the CDS.

\section{Appendix B: MUSE/NS sources}

Table B. 1 lists the properties of the 66 Ly $\alpha$ emitters found by performing our blind search.

Table B.1. Blind search detected high-redshift $(z \geq 2.9)$ Ly $\alpha$ emitting sources in the COSMOS/MUSE GTO field.

\begin{tabular}{|c|c|c|c|c|c|c|c|c|c|}
\hline $\begin{array}{l}\text { ObjID } \\
\text { no. }\end{array}$ & RA & Dec & $z_{\text {spec }}$ & QF & $\begin{array}{c}\text { Line center (Main) } \\
{[\AA]}\end{array}$ & $\begin{array}{c}\text { Line center (Sec.) } \\
{[\AA]}\end{array}$ & $\begin{array}{l}\text { Line profile } \\
{[\mathrm{SP}, \mathrm{BB}, \mathrm{RT}]}\end{array}$ & $\begin{array}{c}F_{\text {fit }} \\
{\left[10^{-18} \mathrm{erg} \mathrm{s}^{-1} \mathrm{~cm}^{-2}\right]}\end{array}$ & $\begin{array}{c}L_{\text {fit }} \\
{\left[10^{42} \mathrm{erg} \mathrm{s}^{-1}\right]}\end{array}$ \\
\hline NS1 & 150.0956195 & 2.207896860 & 3.7137 & 2 & $5730.5 \pm 0.3$ & - & SP & $18.96 \pm 1.83$ & $2.55 \pm 0.25$ \\
\hline NS3 & 150.0890289 & 2.202755060 & 4.0670 & 9 & $6160.0 \pm 0.4$ & - & SP & $13.39 \pm 1.85$ & $2.23 \pm 0.31$ \\
\hline NS4 & 150.0902794 & 2.208985301 & 4.0681 & 3 & $6160.8 \pm 0.3$ & $6163.4 \pm 0.9$ & RT & $22.03 \pm 2.25$ & $3.67 \pm 0.38$ \\
\hline NS6 & 150.1064723 & 2.205999095 & 3.1468 & 9 & $5042.2 \pm 0.3$ & $5035.3 \pm 0.5$ & $\mathrm{BB}$ & $14.57 \pm 1.86$ & $1.32 \pm 0.17$ \\
\hline NS7 & 150.1062366 & 2.202861769 & 3.6407 & 3 & $5641.9 \pm 0.4$ & $5646.8 \pm 2.1$ & RT & $24.50 \pm 5.05$ & $3.13 \pm 0.65$ \\
\hline NS8 & 150.1107880 & 2.196746174 & 4.9088 & 9 & $7182.3 \pm 0.4$ & - & SP & $15.64 \pm 1.65$ & $4.06 \pm 0.43$ \\
\hline NS10 & 150.1271781 & 2.193819620 & 4.5575 & 3 & $6756.1 \pm 0.3$ & $6759.7 \pm 0.9$ & $\mathrm{RT}$ & $26.69 \pm 2.32$ & $5.82 \pm 0.51$ \\
\hline NS15 & 150.1631312 & 2.200003868 & 3.1316 & 9 & $5022.0 \pm 0.5$ & - & SP & $13.49 \pm 2.33$ & $1.20 \pm 0.21$ \\
\hline NS16 & 150.1611895 & 2.206540221 & 3.6455 & 9 & $5645.4 \pm 0.3$ & $5648.9 \pm 1.1$ & RT & $22.51 \pm 3.12$ & $2.89 \pm 0.40$ \\
\hline NS19 & 150.1766375 & 2.200547905 & 3.1329 & 3 & $5024.4 \pm 0.4$ & $5026.9 \pm 2.1$ & RT & $37.82 \pm 7.11$ & $3.37 \pm 0.63$ \\
\hline NS20 & 150.1804873 & 2.207906097 & 3.9656 & 2 & $6036.8 \pm 0.2$ & - & SP & $17.42 \pm 1.30$ & $2.74 \pm 0.20$ \\
\hline NS21 & 150.1897355 & 2.204086497 & 4.0641 & 3 & $6156.4 \pm 0.2$ & - & SP & $23.91 \pm 1.86$ & $3.98 \pm 0.31$ \\
\hline NS22 & 150.1819584 & 2.196144730 & 4.3955 & 9 & $6559.7 \pm 0.4$ & $6563.7 \pm 3.8$ & $\mathrm{RT}$ & $7.04 \pm 3.56$ & $1.41 \pm 0.71$ \\
\hline NS23 & 150.1763508 & 2.194987214 & 4.4405 & 3 & $6612.7 \pm 0.3$ & $6615.4 \pm 0.6$ & RT & $12.50 \pm 1.14$ & $2.56 \pm 0.23$ \\
\hline $\mathrm{NS} 24$ & 150.1871556 & 2.205024286 & 5.0682 & 2 & $7377.7 \pm 0.3$ & - & SP & $8.26 \pm 1.17$ & $2.32 \pm 0.33$ \\
\hline NS27 & 150.0900671 & 2.221593035 & 3.2476 & 3 & $5163.4 \pm 0.5$ & - & SP & $22.50 \pm 2.82$ & $2.19 \pm 0.27$ \\
\hline NS28 & 150.0938165 & 2.212022447 & 3.3896 & 3 & $5336.1 \pm 0.2$ & $5338.3 \pm 1.0$ & RT & $36.35 \pm 4.19$ & $3.92 \pm 0.45$ \\
\hline NS29 & 150.0967870 & 2.210240788 & 3.4315 & 9 & $5387.0 \pm 1.2$ & $5387.4 \pm 0.3$ & RT & $11.21 \pm 2.80$ & $1.24 \pm 0.31$ \\
\hline NS30 & 150.0974611 & 2.210715291 & 3.7856 & 3 & $5818.2 \pm 0.2$ & $5823.5 \pm 0.5$ & RT & $42.11 \pm 3.16$ & $5.92 \pm 0.44$ \\
\hline NS32 & 150.0859870 & 2.224398122 & 4.2863 & 3 & $6426.5 \pm 0.2$ & - & SP & $18.42 \pm 1.40$ & $3.48 \pm 0.26$ \\
\hline NS34 & 150.0870797 & 2.216011780 & 4.9009 & 9 & $7172.5 \pm 0.6$ & $7177.7 \pm 1.3$ & RT & $8.61 \pm 1.52$ & $2.41 \pm 0.43$ \\
\hline NS35 & 150.0968042 & 2.219972452 & 4.9027 & 9 & $7175.7 \pm 0.4$ & - & SP & $15.66 \pm 1.53$ & $4.06 \pm 0.40$ \\
\hline NS36 & 150.0850979 & 2.213391448 & 5.0266 & 9 & $7324.7 \pm 0.3$ & $7327.8 \pm 0.6$ & RT & $18.20 \pm 1.60$ & $5.00 \pm 0.44$ \\
\hline NS40 & 150.1027830 & 2.218819424 & 3.6257 & 9 & $5622.8 \pm 0.2$ & - & SP & $17.26 \pm 2.44$ & $2.19 \pm 0.31$ \\
\hline NS41 & 150.1009535 & 2.213916903 & 3.8283 & 9 & $5870.8 \pm 0.5$ & - & SP & $22.61 \pm 3.47$ & $3.27 \pm 0.50$ \\
\hline NS42 & 150.1045699 & 2.221037671 & 5.2998 & 2 & $7659.2 \pm 0.3$ & $7666.1 \pm 2.8$ & RT & $20.27 \pm 3.15$ & $6.31 \pm 0.98$ \\
\hline NS46 & 150.1273378 & 2.214692674 & 3.0943 & 3 & $4977.4 \pm 0.3$ & - & SP & $33.23 \pm 3.75$ & $2.88 \pm 0.33$ \\
\hline NS48 & 150.1282981 & 2.211522251 & 3.3210 & 9 & $5252.9 \pm 0.4$ & - & SP & $13.47 \pm 2.13$ & $1.38 \pm 0.22$ \\
\hline NS49 & 150.1236488 & 2.221830819 & 4.2820 & 9 & $6420.5 \pm 0.3$ & $6425.5 \pm 2.0$ & RT & $10.05 \pm 1.88$ & $1.89 \pm 0.35$ \\
\hline NS53 & 150.1311033 & 2.221893546 & 3.2790 & 9 & $5202.1 \pm 0.2$ & - & SP & $11.75 \pm 1.37$ & $1.17 \pm 0.14$ \\
\hline NS54 & 150.1444920 & 2.210705671 & 4.1055 & 9 & $6206.9 \pm 0.3$ & - & SP & $10.56 \pm 1.28$ & $1.80 \pm 0.22$ \\
\hline NS55 & 150.1331736 & 2.224723824 & 4.5402 & 9 & $6735.4 \pm 0.3$ & $6738.3 \pm 0.9$ & RT & $7.16 \pm 1.42$ & $1.55 \pm 0.31$ \\
\hline NS56 & 150.1355132 & 2.212112631 & 5.4193 & 9 & $7804.4 \pm 0.2$ & - & SP & $6.95 \pm 0.94$ & $2.28 \pm 0.31$ \\
\hline NS58 & 150.1442147 & 2.221428993 & 5.8863 & 9 & $8372.0 \pm 0.3$ & - & SP & $14.19 \pm 1.30$ & $5.65 \pm 0.52$ \\
\hline NS61 & 150.1572317 & 2.209132493 & 3.8550 & 9 & $5901.6 \pm 0.7$ & $5902.4 \pm 0.8$ & RT & $18.30 \pm 2.67$ & $2.69 \pm 0.39$ \\
\hline
\end{tabular}

Notes. As in Table 1, the ID, position on the sky, measured redshift, and quality flag are displayed. Also listed is the information on the Ly $\alpha$ flux and luminosity measurement. The labels on the line profile refer to the single peak (SP), blue bump (BB), or red tail (RT). 
G. Rosani et al.: Bright Lyman- $\alpha$ emitters among Spitzer SMUVS galaxies in the MUSE/COSMOS field

Table B.1. continued.

\begin{tabular}{|c|c|c|c|c|c|c|c|c|c|}
\hline $\begin{array}{l}\text { ObjID } \\
\text { no. }\end{array}$ & RA & Dec & $z_{\text {spec }}$ & QF & $\begin{array}{c}\text { Line center (Main) } \\
[\AA]]\end{array}$ & $\begin{array}{c}\text { Line center (Sec.) } \\
[\AA]]\end{array}$ & $\begin{array}{l}\text { Line profile } \\
\text { [SP,BB,RT] }\end{array}$ & $\begin{array}{c}F_{\text {fit }} \\
{\left[10^{-18} \mathrm{erg} \mathrm{s}^{-1} \mathrm{~cm}^{-2}\right]}\end{array}$ & $\begin{array}{c}L_{\mathrm{fit}} \\
{\left[10^{42} \mathrm{erg} \mathrm{s}^{-1}\right]} \\
\end{array}$ \\
\hline NS62 & 150.1750090 & 2.212290512 & 2.9695 & 3 & $4825.8 \pm 0.2$ & - & SP & $14.97 \pm 1.80$ & $1.17 \pm 0.14$ \\
\hline NS64 & 150.1644704 & 2.214284122 & 3.0976 & 9 & $4981.4 \pm 0.4$ & $4985.1 \pm 4.8$ & RT & $14.44 \pm 7.93$ & $1.25 \pm 0.69$ \\
\hline NS65 & 150.1617505 & 2.210353565 & 3.4902 & 9 & $5458.4 \pm 0.5$ & - & SP & $8.10 \pm 1.59$ & $0.94 \pm 0.18$ \\
\hline NS67 & 150.1634469 & 2.210140164 & 3.8656 & 3 & $5914.6 \pm 0.3$ & $5919.6 \pm 0.4$ & RT & $16.96 \pm 1.57$ & $2.51 \pm 0.23$ \\
\hline NS68 & 150.1674625 & 2.215432552 & 4.4342 & 9 & $6606.2 \pm 0.8$ & - & SP & $10.62 \pm 1.67$ & $2.17 \pm 0.34$ \\
\hline NS72 & 150.1818099 & 2.215637886 & 5.2455 & 9 & $7592.6 \pm 0.3$ & - & SP & $25.86 \pm 2.79$ & $7.87 \pm 0.85$ \\
\hline NS73 & 150.0847432 & 2.233357253 & 3.2413 & 3 & $5155.9 \pm 0.2$ & $5157.3 \pm 1.1$ & RT & $54.08 \pm 4.63$ & $5.24 \pm 0.45$ \\
\hline NS74 & 150.0865742 & 2.227631189 & 3.4529 & 9 & $5413.2 \pm 0.2$ & - & SP & $14.25 \pm 1.57$ & $1.61 \pm 0.18$ \\
\hline NS75 & 150.0946790 & 2.230024846 & 4.0667 & 3 & $6160.4 \pm 0.3$ & - & SP & $13.55 \pm 1.50$ & $2.26 \pm 0.25$ \\
\hline NS78 & 150.0964429 & 2.229859080 & 5.7850 & 9 & $8249.5 \pm 0.3$ & - & SP & $8.59 \pm 1.35$ & $3.29 \pm 0.52$ \\
\hline NS79 & 150.1131469 & 2.230677797 & 3.2805 & 9 & $5203.0 \pm 0.5$ & - & SP & $56.12 \pm 6.08$ & $5.60 \pm 0.61$ \\
\hline NS80 & 150.1237063 & 2.229857749 & 3.4468 & 9 & $5406.0 \pm 0.2$ & - & SP & $20.57 \pm 2.49$ & $2.31 \pm 0.28$ \\
\hline NS81 & 150.1244038 & 2.231267693 & 3.9679 & 3 & $6039.8 \pm 0.3$ & - & SP & $24.76 \pm 3.44$ & $3.89 \pm 0.54$ \\
\hline NS82 & 150.1390775 & 2.235081324 & 3.1188 & 3 & $5007.3 \pm 0.2$ & $4996.1 \pm 1.1$ & $\mathrm{BB}$ & $75.78 \pm 4.98$ & $6.70 \pm 0.44$ \\
\hline NS83 & 150.1438608 & 2.231322324 & 3.2779 & 9 & $5199.8 \pm 0.3$ & - & SP & $4.65 \pm 1.33$ & $0.46 \pm 0.13$ \\
\hline NS85 & 150.1599066 & 2.230670888 & 3.3592 & 9 & $5299.7 \pm 0.3$ & $5306.4 \pm 0.5$ & RT & $15.30 \pm 1.79$ & $1.61 \pm 0.19$ \\
\hline NS86 & 150.1587096 & 2.240617386 & 3.6127 & 9 & $5607.9 \pm 0.6$ & - & SP & $53.24 \pm 7.24$ & $6.68 \pm 0.91$ \\
\hline NS87 & 150.1509930 & 2.226192297 & 3.6782 & 3 & $5687.1 \pm 0.7$ & $5690.1 \pm 1.3$ & RT & $19.96 \pm 3.92$ & $2.62 \pm 0.51$ \\
\hline NS88 & 150.1525301 & 2.238594726 & 4.5186 & 3 & $6708.8 \pm 0.3$ & - & SP & $7.91 \pm 0.87$ & $1.69 \pm 0.19$ \\
\hline NS91 & 150.1864893 & 2.234161180 & 3.6828 & 9 & $5692.3 \pm 0.9$ & - & SP & $6.49 \pm 1.89$ & $0.86 \pm 0.25$ \\
\hline NS92 & 150.1805399 & 2.235132627 & 3.8673 & 3 & $5917.2 \pm 0.3$ & - & SP & $12.74 \pm 1.69$ & $1.88 \pm 0.25$ \\
\hline NS97 & 150.1916706 & 2.235040683 & 5.9697 & 9 & $8474.4 \pm 1.2$ & - & SP & $13.06 \pm 2.93$ & $5.37 \pm 1.21$ \\
\hline NS98 & 150.1057122 & 2.328948835 & 3.2080 & 9 & $5116.1 \pm 0.4$ & - & SP & $11.11 \pm 1.97$ & $1.05 \pm 0.19$ \\
\hline NS99 & 150.1192540 & 2.333246831 & 3.0062 & 9 & $4870.6 \pm 0.4$ & - & SP & $22.58 \pm 3.04$ & $1.82 \pm 0.25$ \\
\hline NS100 & 150.1183659 & 2.321809003 & 3.2649 & 3 & $5184.9 \pm 0.3$ & - & SP & $19.95 \pm 2.35$ & $1.97 \pm 0.23$ \\
\hline NS101 & 150.1247395 & 2.321276301 & 3.4198 & 9 & $5373.7 \pm 0.6$ & - & SP & $14.39 \pm 2.53$ & $1.58 \pm 0.28$ \\
\hline NS102 & 150.1146837 & 2.323507763 & 3.4973 & 2 & $5469.1 \pm 0.5$ & - & SP & $13.88 \pm 2.10$ & $1.61 \pm 0.24$ \\
\hline NS103 & 150.1152667 & 2.328748203 & 3.9439 & 9 & $6010.5 \pm 0.3$ & - & SP & $21.10 \pm 2.37$ & $3.27 \pm 0.37$ \\
\hline NS105 & 150.1280974 & 2.320155687 & 4.4369 & 9 & $6609.6 \pm 0.3$ & - & SP & $11.86 \pm 1.39$ & $2.43 \pm 0.29$ \\
\hline NS106 & 150.1137025 & 2.323197438 & 4.4971 & 9 & $6683.0 \pm 0.4$ & - & SP & $12.47 \pm 1.84$ & $2.64 \pm 0.39$ \\
\hline NS107 & 150.1216946 & 2.320388333 & 4.5356 & 9 & $6729.5 \pm 0.2$ & - & SP & $9.03 \pm 1.17$ & $1.95 \pm 0.25$ \\
\hline
\end{tabular}

